\title{
Topology optimization of flexoelectric composites using computational homogenization
}

\author{
X. Chen ${ }^{\mathrm{a}, \mathrm{b}}$, J. Yvonnet ${ }^{\mathrm{b}, *}$, S. Yao ${ }^{\mathrm{a}}$, H.S. Park ${ }^{\mathrm{c}}$ \\ ${ }^{a}$ Key Laboratory of Traffic Safety on Track, Ministry of Education, School of Traffic \& Transportation \\ Engineering, Central South University, Changsha 410075, China \\ ${ }^{b}$ MSME, Univ Gustave Eiffel, CNRS UMR 8208, F-77474 Marne-la-Vallée, France \\ ${ }^{c}$ Department of Mechanical Engineering, Boston University, Boston, Massachusetts 02215, US
}

\begin{abstract}
We present a topology optimization framework to design periodic composites comprised of piezoelectric constituents that exhibit large flexoelectric constants. The novelty of the approach is that it leverages a representative volume element (RVE)-based computational homogenization approach that enables the analysis of periodic composites where the characteristic dimensions of the microstructure are significantly smaller than those of the structure, and as such requires only the optimization of a single RVE rather than that of the entire structure. We utilize this approach to analyze the enhancement in flexoelectric constants that can be achieved in different types of PZT-based composites, including hard-hard (PZT-PZT), and hard-soft (PZT-polymer composite, and porous PZT) structures. In all cases, significant enhancements are observed, with improvements between 2 and 15 times those of a naive guess, with some designs reaching a factor of one order of magnitude larger than BTO. We identify different mechanisms governing the enhanced electromechanical couplings, which can arise either from an enhancement of effective piezoelectricity in the RVE for PZT-PZT composites, or from a more subtle interplay involving the enhancement of effective piezoelectric and dielectric properties coupled with a reduction in mechanical compliance for PZT-polymer and porous PZT RVEs.
\end{abstract}

Keywords: Flexoelectricity, topology optimization, Computational homogenization,

\footnotetext{
${ }^{*}$ Corresponding author

Email address: julien. yvonnet@univ-par is-est.fr (J. Yvonnet)
} 
multi scale methods

\section{Introduction}

Flexoelectricity is the property of an insulating material to polarize when subjected to strain gradient (inhomogeneous deformation). Even though flexoelectric effects are much larger in ferroelectric materials [1] and complex oxide ceramics [2, 3, 4, 5], the flexoelectricity of several polymers has recently been investigated in [6, 7] as well as in biological membranes in [8]. Kogan [9] formulated the first phenomenological theory of flexelectricity and estimated the range of values for flexoelectric coefficients. Tagantsev [10,11] developed a microscopic theory for the bulk contributions, using the rigid-ion approximation as well as a phenomenological description.

The flexoelectric effect has been widely studied in recent years due to potential applications in soft robotics, energy harvesters, stretchable electronics, sensors and actuators as additional apparent piezoelectric effects can be induced. The fourth-order partial differential equations of flexoelectric coupling systems have been approached with analytical solutions on simplifying assumptions and simple structural geometries [12, 13, 14, 15, 16, 17]. Recently, a computational framework to calculate the flexoelectric effect in dielectric solids using a meshfree approach was proposed [18, 19, 20]. A B-spline approach has been proposed in [21].

Reviews and discussions on flexoelectricity can be found e.g. in [22, 7, 23, 24, 25, [26, 27].

An ongoing challenge for exploiting flexoelectricity is that the effect is usually quite low in homogeneous (bulk) materials. The flexoelectric constants can be relatively high in stiff ceramics such as $\mathrm{BaTiO}_{3}$ (BTO), while it is typically negligible in soft materials such as polymers. There have been various approaches to enhancing the flexoelectric constants of solids. One approach has been to consider electrets, i.e. by embedding fixed charges in the material [28]. Another approach has been to use architected materials comprised of piezoelectric phases [29], where the effective flexoelectric properties of the piezoelectric composites are obtained using homogenization [30, 31, 32, 33, 34]. More recently, topology optimization [35, 36, 37, 38, 39, 
40, 41, 42], which has a lengthy and successful history of determining, using inverse homogenization methods [43] the optimal material distribution to maximize a specific property [44, 43, 45, 46], has been applied to increase the apparent flexoelectricity and energy conversion in piezoelectric structures [47, 48, 49, 50].

However, to fully exploit the possibility of designing structures to maximize their flexoelectric properties, advances beyond analytical homogenization theories or computational topology optimization of macroscale structures are required. In particular, what has not been achieved to-date is a reliable approach to tailoring the microstructure of a composite to enhance the flexoelectric properties, particularly if the microstructure exists at a scale that is significantly smaller than that of the structure.

One appealing tool for this task is the use of Topology Optimization (TO). Firstly proposed by Bendsøe and Kikuchi [51], TO has since been applied to a large variety of problems in engineering. TO methods can be broadly categorized into three families: (a) the Solid Isotropic Material with Penalization (SIMP) method [52, 53, 54], (b) the level set method [55, 56], and (c) the Evolutionary Structural Optimization (ESO) method [57]. Review of these methods can be found in [58, 59, 60, 61]). A comparison review on these techniques, with advantages and drawbacks, can be found in [40]. A survey on the applications of TO to a broad variety of problems including mechanical and thermal loads of structures, fluid flow, dynamics, acoustics and biomechanicscan be found in [61]. Recently, Ganghoffer et al. [62] used TO together with the concept of topological derivative for designing auxetic microstructures exhibiting strain gradient behavior.

In the present work, we propose a topology optimization framework to design periodic composites comprised of piezoelectric constituents that exhibit large flexoelectric constants. The approach leverages a recently-developed computational homogenization framework for effective flexoelectric materials [63] that enables the estimation of the (apparent) effective flexoelectric properties of a periodic composite made of piezoelectric phases. The different associated sensitivity expression are derived in this context and a SIMP (Solid Isotropic Material with Penalization) topology optimization framework is developed. A novel aspect of this approach is that it allows us to restrict the analysis to a single representative volume element (RVE) that describes the 
microstructure, and importantly does not require the optimization of the fully detailed structure. This presents significant advantages when there is scale separation, i.e. when the characteristic dimensions of the heterogeneities are negligible as compared to those of the structure. We utilize this approach to analyze the enhancement in flexoelectric constants that can be achieved in different types of PZT-based composites, including hard-hard (PZT-PZT), and hard-soft (PZT-polymer composite, and porous PZT) structures. In all cases, significant enhancements are observed, with improvements between 2 and 15 times those of a naive guess, with some designs reaching a factor of one order of magnitude larger than BTO. We identify different mechanisms governing the enhanced electromechanical couplings, which can arise either from an enhancement of effective piezoelectricity in the RVE for PZT-PZT composites, or from a more subtle interplay involving the enhancement of effective piezoelectric and dielectric properties coupled with a reduction in mechanical compliance for PZT-polymer and porous PZT RVEs.

The reminder of this paper is as follows. In Section 2 , we introduce the homogenization theory which is employed in the optimization process. The topology optimization problem for maximizing the effective flexoelectric coefficients of the material is provided in Section 4 Section 5 presents numerical examples to investigate the potential of the approach for several representative cases, including PZT/PZT composites, PZT/polymer composites and voided PZT materials. Conclusion and perspectives are provided in Section 6

\section{Homogenization framework}

In this section, we review the computational homogenization framework proposed in our previous work [63] for piezoelectric composites with effective flexoelectric behavior.

\subsection{Micro scale problem}

We consider a periodic composite (see Fig. 11 a)) assumed to be characterized by a Representative Volume Element (RVE) (see fig 1 (c)). The RVE is defined in a domain 


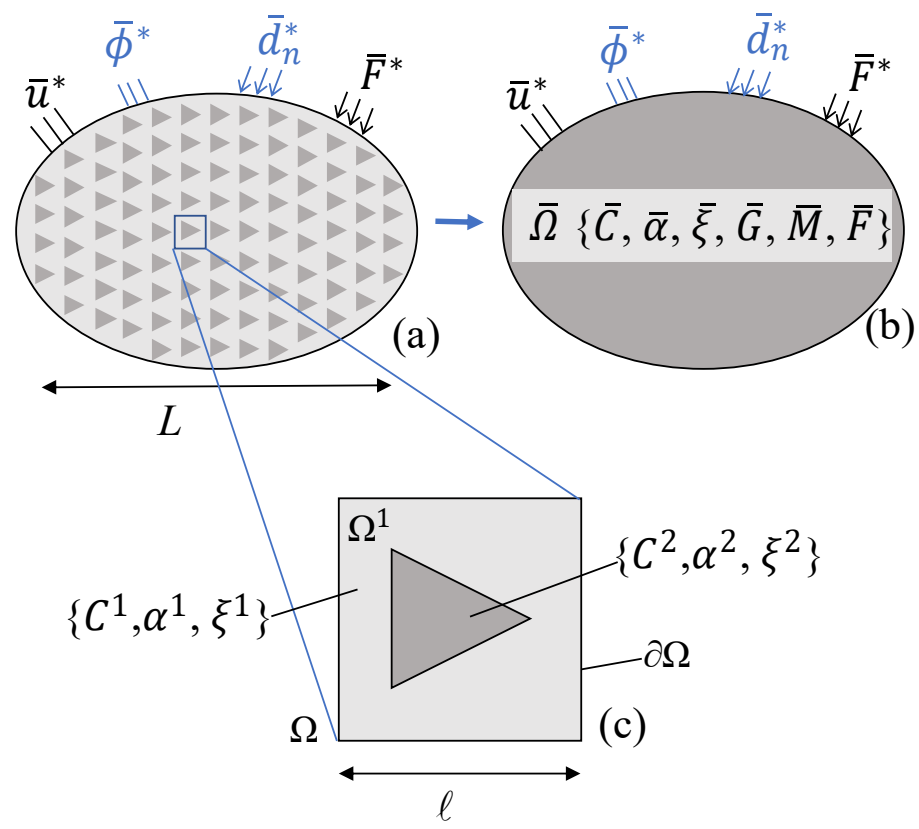

Figure 1: (a) Periodic heterogeneous structure; (b) Equivalent piezo-flexoelectric homogeneous structure;(c) RVE model

$\Omega \in \mathbb{R}^{d}$ whose external boundary is denoted by $\partial \Omega$. The characteristic size of the RVE is $\ell$. The RVE is assumed to be subjected to three homogeneous fields: a strain $\bar{\varepsilon}$, a strain gradient $\nabla \bar{\varepsilon}$ and an electric field $\overline{\mathbf{E}}$. The different phases of the RVE are assumed to be linear piezoelectric and characterized by an elastic tensor $\mathbb{C}^{k}$, a dielectric tensor $\alpha^{k}$ and a piezoelectric tensor $\mathscr{E}^{k}, k=1, \ldots, N_{p}$, with $N_{p}$ the number of phases.

The energy density function (electrical enthalpy density) of a piezoelectric material is defined by:

$$
W=\frac{1}{2} \varepsilon(\mathbf{x}): \mathbb{C}(\mathbf{x}): \varepsilon(\mathbf{x})-\mathbf{E}(\mathbf{x}) \cdot \mathscr{E}(\mathbf{x}): \varepsilon(\mathbf{x})-\frac{1}{2} \mathbf{E}(\mathbf{x}) \cdot \alpha(\mathbf{x}) \cdot \mathbf{E}(\mathbf{x})
$$

where $\mathbb{C}$ is the fourth-order elastic tensor, $\alpha$ is the second-order dielectric tensor, $\mathscr{E}$ is the third-order piezoelectric tensor and $\mathbf{x}$ denotes coordinates. Then the Cauchy stress 
$\sigma$ and the electric displacement $\mathbf{d}$ are defined by:

$$
\begin{aligned}
& \boldsymbol{\sigma}(\mathbf{x})=\frac{\partial W}{\partial \varepsilon}=\mathbb{C}(\mathbf{x}): \varepsilon(\mathbf{x})-\mathscr{E}(\mathbf{x}) \cdot \mathbf{E}(\mathbf{x}) \\
& \mathbf{d}(\mathbf{x})=-\frac{\partial W}{\partial \mathbf{E}}=\mathscr{E}(\mathbf{x}): \varepsilon(\mathbf{x})+\boldsymbol{\alpha}(\mathbf{x}) \cdot E(\mathbf{x})
\end{aligned}
$$

The effective electric field can be computed by prescribing the following boundary conditions over the RVE:

$$
\phi(\mathbf{x})=-\overline{\mathbf{E}} \cdot \mathbf{x}+\tilde{\phi}(\mathbf{x}) \text { on } \partial \Omega
$$

where $\tilde{\phi}(\mathbf{x})$ is either zero or a periodic fluctuation on $\partial \Omega$. A quadratic boundary condition (QBC) has been introduced to prescribe an effective strain and strain gradient 64 , 65]:

$$
\mathbf{u}(\mathbf{x})=\bar{\varepsilon} \cdot \mathbf{x}+\frac{1}{2} \overline{\mathscr{G}}: \mathbf{x} \otimes \mathbf{x}+\tilde{\mathbf{u}}(\mathbf{x}) \text { on } \partial \Omega
$$

where

$$
\overline{\mathscr{G}}_{i j k}=\overline{\nabla \varepsilon}_{i j k}+\overline{\nabla \varepsilon}_{i k j}-\overline{\nabla \varepsilon}_{j k i}
$$

and $\tilde{\mathbf{u}}(\mathbf{x})$ is either zero or periodic on $\partial \Omega$. However, quadratic boundary conditions alone can induce spurious gradient terms and a non-convergence of the higher order effective coefficients with respect to RVE characteristic size. These issues can be eliminated by introducing body forces to enforce a constant strain gradient within the RVE when the material is homogeneous[66].

Then, local equations are substituted by

$$
\begin{aligned}
& \nabla \cdot \boldsymbol{\sigma}(\mathbf{u}(\mathbf{x}))=f(\overline{\nabla \varepsilon}) \mathbf{x} \in \Omega \\
& \nabla \cdot \mathbf{d}(\mathbf{x})=r(\overline{\nabla \varepsilon}) \mathbf{x} \in \Omega
\end{aligned}
$$

where

$$
\begin{aligned}
& f_{i}=\mathbb{C}_{i j k l}^{0} \overline{\nabla \varepsilon}_{k l j} \\
& r=\mathscr{E}_{i j k}^{0} \overline{\nabla \varepsilon}_{j k i}
\end{aligned}
$$


are body forces added to remove spurious fluctuations in the case of homogeneous RVEs (see [66] and [63] for more details). Above, $\sigma$ and $\mathbf{d}$ are given by Eqs. (2), (3).

Eqs. (7)-(8) are completed with the boundary conditions (4)-(5).

Solving the linear localization problem (7)-(8)-(4)-(5) by using the superposition principle, the local strain field $\varepsilon(\mathbf{x})$ and the local electric field $\mathbf{E}(\mathbf{x})$ can be obtained as:

$$
\begin{aligned}
& \varepsilon(\mathbf{x})=\mathbb{A}^{0}(\mathbf{x}): \bar{\varepsilon}+\mathscr{B}^{0}(\mathbf{x}) \cdot \overline{\mathbf{E}}+\tilde{\mathbb{A}}^{1}(\mathbf{x}) \vdots \overline{\nabla \varepsilon} \\
& \mathbf{E}(\mathbf{x})=\mathscr{D}^{0}(\mathbf{x}): \bar{\varepsilon}+\mathbf{h}^{0}(\mathbf{x}) \cdot \overline{\mathbf{E}}+\tilde{\mathbb{D}}^{1}(\mathbf{x}): \overline{\nabla \varepsilon}
\end{aligned}
$$

where

$$
\begin{aligned}
& \tilde{\mathbb{A}}^{1}(\mathbf{x})=\mathbb{A}^{1}(\mathbf{x})-\mathbb{A}^{0}(\mathbf{x}) \otimes \mathbf{x} \\
& \tilde{\mathbb{D}}^{1}(\mathbf{x})=\mathbb{D}^{1}(\mathbf{x})-\mathbb{D}^{0}(\mathbf{x}) \otimes \mathbf{x}
\end{aligned}
$$

are corrected terms to remove local spurious fluctuations in the case of homogeneous RVEs 66, 63].

The strain solutions are calculated by:

i) $\mathbb{A}^{0}(\mathbf{x}), \mathscr{B}^{0}(\mathbf{x})$ and $\mathbb{A}^{1}(\mathbf{x})$ are the strain solution $\varepsilon(\mathbf{x})$ obtained by solving the problems (7)-(8)-(4)-(5) with $\bar{\varepsilon}=\frac{1}{2}\left(\mathbf{e}_{k} \otimes \mathbf{e}_{l}+\mathbf{e}_{l} \otimes \mathbf{e}_{k}\right), \overline{\mathbf{E}}=\mathbf{e}_{k}, \overline{\nabla \varepsilon}=\frac{1}{2}\left(\mathbf{e}_{k} \otimes \mathbf{e}_{l}+\mathbf{e}_{l} \otimes\right.$ $\left.\mathbf{e}_{k}\right) \otimes \mathbf{e}_{m}$, respectively.

ii) $\mathscr{D}^{0}(\mathbf{x}), \mathbf{h}^{0}(\mathbf{x})$ and $\mathbb{D}^{1}(\mathbf{x})$ are the electric field solution $\mathbf{E}(\mathbf{x})$ obtained by solving the problems (7)-(8)-(47)-(5) with $\overline{\nabla \varepsilon}=\frac{1}{2}\left(\mathbf{e}_{j} \otimes \mathbf{e}_{k}+e_{k} \otimes \mathbf{e}_{j}\right), \overline{\mathbf{E}}=\mathbf{e}_{j}, \nabla \bar{\varepsilon}=\frac{1}{2}\left(\mathbf{e}_{j} \otimes\right.$ $\left.\mathbf{e}_{k}+\mathbf{e}_{k} \otimes \mathbf{e}_{j}\right) \otimes \mathbf{e}_{l}$, respectively.

The terms $e_{i}$ are unitary basis vectors.

\section{Finite element discretization of local RVE equation}

In the following, we present the Finite Element discretization for localization problem defined in section 2.1. The 2D plane strain condition is considered. 
The weak form associated with the coupled problem (7)-(8)-(4)-(5) is to find $\mathbf{u} \in$ $\left\{\mathbf{u}=\overline{\mathbf{u}}^{*}\right.$ on $\left.\partial \Omega_{u}, \mathbf{u} \in \mathbf{H}^{1}(\Omega)\right\}$ and $\phi \in\left\{\phi=\bar{\phi}^{*}\right.$ on $\left.\partial \Omega_{\phi}, \phi \in \mathbf{H}^{1}(\Omega)\right\}$ such that 63]

$$
\begin{aligned}
& \int_{\Omega} \nabla(\mathbf{d}) \cdot \delta \phi d \Omega=-\int_{\Omega}\{\overline{\mathscr{E}}: \nabla \bar{\varepsilon} \mathbf{x}\} \cdot \nabla(\delta \phi) d \Omega \\
& \int_{\Omega} \nabla(\boldsymbol{\sigma}): \delta \mathbf{u} d \Omega=\int_{\Omega}\{\overline{\mathbb{C}}: \nabla \bar{\varepsilon} \mathbf{x}\} \cdot \varepsilon(\delta \mathbf{u}) d \Omega
\end{aligned}
$$

for all $\delta \mathbf{u} \in\left\{\delta \mathbf{u}=0\right.$ on $\left.\partial \Omega_{u}, \delta \mathbf{u} \in \mathbf{H}^{1}(\Omega)\right\}$ and $\delta \phi \in\left\{\delta \phi=0\right.$ on $\left.\partial \Omega_{\phi}, \delta \phi \in \mathbf{H}^{1}(\Omega)\right\}$. Substituting Eq. (2) and (3) into Eq. (15) and (16) yields:

$$
\begin{aligned}
& \int_{\Omega}(\mathscr{E}: \varepsilon(\mathbf{u})+\boldsymbol{\alpha} \cdot \mathbf{E}(\phi)) \cdot \nabla(\boldsymbol{\delta} \phi) d \Omega=-\int_{\Omega}\{\overline{\mathscr{E}}: \overline{\nabla \varepsilon} \mathbf{x}\} \cdot \nabla(\boldsymbol{\delta} \phi) d \Omega \\
& \int_{\Omega}\left(\mathbb{C}: \varepsilon(\mathbf{u})-\mathscr{E}^{T} \cdot \mathbf{E}(\phi)\right): \varepsilon(\delta \mathbf{u}) d \Omega=\int_{\Omega}\{\overline{\mathbb{C}}: \bar{\nabla} \varepsilon \mathbf{x}\} \cdot \varepsilon(\delta \mathbf{u}) d \Omega
\end{aligned}
$$

We adopt the same finite element discretization for the approximation of the displacement field $\mathbf{u}$ and of the electric field $\phi$. Using 8-node element, the two finite element approximate field $\left(\mathbf{u}^{h}, \phi^{h}\right)$ can be expressed as:

$$
\begin{aligned}
& \mathbf{u}^{h}(\mathbf{x})=\mathbf{N}_{u}(\mathbf{x}) \mathbf{u}_{\mathbf{i}} ; \phi^{h}(\mathbf{x})=\mathbf{N}_{\phi}(\mathbf{x}) \phi_{\boldsymbol{i}} \\
& \delta \mathbf{u}^{h}(\mathbf{x})=\mathbf{N}_{u}(\mathbf{x}) \delta \mathbf{u}_{\mathbf{i}} ; \delta \phi^{h}(\mathbf{x})=\mathbf{N}_{\phi}(\mathbf{x}) \boldsymbol{\delta} \phi_{\boldsymbol{i}}
\end{aligned}
$$

and their derivatives as,

$$
\begin{aligned}
& \nabla \mathbf{u}^{h}(\mathbf{x})=\mathbf{B}_{u}(\mathbf{x}) \mathbf{u}_{\mathbf{i}} ; \nabla \phi^{h}(\mathbf{x})=\mathbf{B}_{\phi}(\mathbf{x}) \phi_{i} \\
& \nabla \delta \mathbf{u}^{h}(\mathbf{x})=\mathbf{B}_{u}(\mathbf{x}) \delta \mathbf{u}_{\mathbf{i}} ; \nabla \delta \phi^{h}(\mathbf{x})=\mathbf{B}_{\phi}(\mathbf{x}) \delta \phi_{i}
\end{aligned}
$$

By substituting the above discrete approximation in $\mathrm{Eq}(17)$ and $(18)$, we obtain the linear system of coupling equations:

$$
\left[\begin{array}{cc}
\mathbf{K}_{\phi \phi} & \mathbf{K}_{\phi u} \\
-\mathbf{K}_{u \phi} & \mathbf{K}_{u u}
\end{array}\right]\left[\begin{array}{c}
\boldsymbol{\phi} \\
\boldsymbol{u}
\end{array}\right]=\left[\begin{array}{c}
\mathbf{F}_{\phi} \\
\mathbf{F}_{u}
\end{array}\right]
$$


with

$$
\begin{aligned}
& \mathbf{K}_{\phi \phi}=\int_{\Omega}\left(\mathbf{B}_{\phi}\right)^{T}[\boldsymbol{\alpha}] \mathbf{B}_{\phi} d \Omega, \\
& \mathbf{K}_{\phi u}=-\int_{\Omega}\left(\mathbf{B}_{\phi}\right)^{T}[\mathscr{E}] \mathbf{B}_{u} d \Omega, \\
& \mathbf{K}_{u u}=\int_{\Omega}\left(\mathbf{B}_{u}\right)^{T}[\mathbb{C}] \mathbf{B}_{u} d \Omega, \\
& \mathbf{F}_{\phi}=\int_{\Omega}\left(\mathbf{B}_{\phi}\right)^{T}[\mathscr{E}][\boldsymbol{\kappa}] d \Omega, \\
& \mathbf{F}_{u}=\int_{\Omega}\left(\mathbf{B}_{u}\right)^{T}[\mathbb{C}][\boldsymbol{\kappa}] d \Omega,
\end{aligned}
$$

and

$$
[\kappa]=\left[\begin{array}{c}
x \overline{\nabla \varepsilon}_{111}+y \overline{\nabla \varepsilon}_{112} \\
x \overline{\nabla \varepsilon}_{221}+y \overline{\nabla \varepsilon}_{222} \\
x \overline{\nabla \varepsilon}_{121}+y \overline{\nabla \varepsilon}_{122}
\end{array}\right]
$$

\subsection{Effective piezo-flexoelectric tensors}

In our previous work[63], a general energy density function for an effective piezoflexoelectric material was proposed, extending a Mindlin strain gradient model with electromechanical terms as:

$$
\begin{aligned}
\bar{W}= & \frac{1}{2} \bar{\varepsilon}: \overline{\mathbb{C}}: \bar{\varepsilon}-\frac{1}{2} \overline{\mathbf{E}} \cdot \overline{\boldsymbol{\alpha}} \cdot \mathbf{E}-\overline{\mathbf{E}} \cdot \overline{\mathscr{E}}: \bar{\varepsilon} \\
& +\frac{1}{2} \nabla \bar{\varepsilon}: \overline{\mathbb{G}}: \nabla \bar{\varepsilon}+\overline{\mathbf{E}} \cdot \overline{\mathbb{F}}: \nabla \bar{\varepsilon}+\bar{\varepsilon}: \overline{\mathbb{M}}: \overline{\nabla \varepsilon}
\end{aligned}
$$

where $\overline{\mathbb{G}}$ is the sixth-order effective strain gradient elastic tensor, $\overline{\mathbb{F}}$ is the fourth-order effective flexoelectric tensor coupling electric field and strain gradient, and $\bar{M}$ is a fifthorder effective tensor coupling strain and strain gradient. Above, $(\cdot) \vdots(\cdot)$ denotes triple contraction of indices. Perfect interfaces between different phases are assumed. Note that the above model neglects terms related to electric strain gradient, e.g. associated with the converse flexoelectric gradient (see e.g. [67, 68, 69]). Such extension could be included in the present topology optimization framework and will be the topic of future studies. 
The effective stress tensor $\overline{\boldsymbol{\sigma}}$, effective electric displacement $\overline{\mathbf{d}}$ and effective hyperstress tensor $\overline{\mathscr{S}}$ associated with energy density function 30 are defined as:

$$
\overline{\boldsymbol{\sigma}}=\frac{\partial \bar{W}}{\partial \bar{\varepsilon}}, \quad \overline{\mathbf{d}}=-\frac{\partial \bar{W}}{\partial \overline{\mathbf{E}}}, \quad \overline{\mathscr{S}}=\frac{\partial \bar{W}}{\partial \overline{\nabla \varepsilon}}
$$

Taking the spatial average of (1) we obtain:

$$
\bar{W}=\frac{1}{2}\langle\varepsilon(\mathbf{x}): \mathbb{C}(\mathbf{x}): \varepsilon(\mathbf{x})\rangle-\langle\mathbf{E}(\mathbf{x}) \cdot \mathscr{E}(\mathbf{x}): \varepsilon(\mathbf{x})\rangle-\frac{1}{2}\langle\mathbf{E}(\mathbf{x}) \cdot \alpha(\mathbf{x}) \cdot \mathbf{E}(\mathbf{x})\rangle
$$

where $\langle\cdot\rangle=\frac{1}{\Omega} \int_{\Omega} \cdot d \Omega$ denotes the volume average over $\Omega$. Then substituting Eq. 11 , and $(12)$ into Eq. (32), and comparing the different terms with Eq. 30 , the effective operators are obtained:

$$
\begin{aligned}
\overline{\mathbb{C}}= & \left\langle\left(\mathbb{A}^{0}(\mathbf{x})\right)^{T}: \mathbb{C}(\mathbf{x}): \mathbb{A}^{0}(\mathbf{x})\right. \\
& \left.-2\left(\mathscr{D}^{0}(\mathbf{x})\right)^{T} \cdot \mathscr{E}(\mathbf{x}): \mathbb{A}^{0}(\mathbf{x})-\left(\mathscr{D}^{0}(\mathbf{x})\right)^{T} \cdot \alpha(\mathbf{x}) \cdot \mathscr{D}^{0}(\mathbf{x})\right\rangle \\
\bar{\alpha}= & \left\langle-\left(\mathscr{B}^{0}(\mathbf{x})\right)^{T}: \mathbb{C}(\mathbf{x}): \mathscr{B}^{0}(\mathbf{x})\right. \\
& \left.+2\left(\mathbf{h}^{0}(\mathbf{x})\right)^{T} \cdot \mathscr{E}(\mathbf{x}): \mathscr{B}^{0}(\mathbf{x})+\left(\mathbf{h}^{0}(\mathbf{x})\right)^{T} \cdot \alpha(\mathbf{x}) \cdot \mathbf{h}^{0}(\mathbf{x})\right\rangle \\
& \overline{\mathscr{E}=} \\
& \left\langle-\left(\mathscr{B}^{0}(\mathbf{x})\right)^{T}: \mathbb{C}(\mathbf{x}): \mathbb{A}^{0}(\mathbf{x})+\left(\mathbf{h}^{0}(\mathbf{x})\right)^{T} \cdot \mathscr{E}(\mathbf{x}): \mathbb{A}^{0}(\mathbf{x})\right. \\
& \left.+\left(\mathscr{B}^{0}(\mathbf{x})\right)^{T}: \mathscr{E}(\mathbf{x}) \cdot \mathscr{D}^{0}(\mathbf{x})+\left(\mathbf{h}^{0}(\mathbf{x})\right)^{T} \cdot \alpha(\mathbf{x}) \cdot \mathscr{D}^{0}(\mathbf{x})\right\rangle \\
& \\
\overline{\mathbb{F}}= & \left\langle\left(\mathscr{B}^{0}(\mathbf{x})\right)^{T}: \mathbb{C}(\mathbf{x}): \tilde{\mathbb{A}}^{1}(\mathbf{x})-\left(\mathbf{h}^{0}(\mathbf{x})\right)^{T} \cdot \mathscr{E}(\mathbf{x}): \tilde{\mathbb{A}}^{1}(\mathbf{x})\right. \\
& \left.-\left(\mathscr{B}^{0}(\mathbf{x})\right)^{T}: \mathscr{E} T(\mathbf{x}) \cdot \tilde{\mathscr{D}}^{1}(\mathbf{x})-\left(\mathbf{h}^{0}(\mathbf{x})\right)^{T} \cdot \alpha(\mathbf{x}) \cdot \tilde{\mathscr{D}}^{1}(\mathbf{x})\right\rangle
\end{aligned}
$$

Note that the expressions for $\bar{M}$ and $\overline{\mathbb{G}}$ in $\underline{30}$, which are not used in the present paper, can be found in [63]. These tensors are only used if we perform structure calculations using the homogenized model (30)-31] (see e.g. [18, 19] for FEM related formulations). The complete expressions for these tensors can be found in [63]. The 
finite element evaluations and matrix forms for the effective tensors are presented in Appendix A The matrix form of the flexoelectric tensor is given in $2 \mathrm{D}$ by:

$$
[\overline{\mathbb{F}}]=\left[\begin{array}{llllll}
\bar{F}_{1111} & \bar{F}_{1221} & \bar{F}_{1122} & \bar{F}_{1222} & \bar{F}_{1112} & \bar{F}_{1121} \\
\bar{F}_{2111} & \bar{F}_{2221} & \bar{F}_{2122} & \bar{F}_{2222} & \bar{F}_{2112} & \bar{F}_{2121}
\end{array}\right]
$$

\subsection{The electromechanical coupling}

A useful parameter to compare different piezoelectric and flexoelectric materials is the electromechanical coupling coefficient denoted as $\mathscr{K}$. The coupling coefficient is a measure of the conversion efficiency between mechanical and electrical energy using the piezoelectric material. It takes the same indices as the piezoelectric coefficient $\mathscr{E}$ and is formulated as [70]

$$
\overline{\mathscr{K}}_{i J}=\frac{\overline{\mathscr{D}}_{i J}}{\sqrt{\overline{\bar{e}}_{i i} \overline{\mathbb{S}}_{J J}}}
$$

with

$$
[\overline{\mathscr{D}}]=[\overline{\mathscr{E}}]:[\overline{\mathbb{C}}]^{-1},[\overline{\mathbf{e}}]=\left([\overline{\mathscr{E}}]:[\overline{\mathbb{C}}]^{-1}:[\overline{\mathscr{E}}]^{T}+[\bar{\alpha}]\right):[\overline{\mathbb{C}}]^{-1}[\overline{\mathbb{S}}]=[\overline{\mathbb{C}}]^{-1}
$$

where $\overline{\mathscr{D}}, \overline{\mathbf{e}}$ and $\overline{\mathbb{S}}$ denote the piezoelectric coefficient, dielectric constant and compliance matrix, respectively.

\section{SIMP topology optimization for flexoelectric composites}

\subsection{Topology optimization problem formulation}

Here we formulate the topology optimization problem to maximize the absolute values of the flexoelectric tensor components in A.6 and (36). First, the periodic unit cell is discretized into $N_{e}$ finite elements which match the mesh used for solving the electromechanical problem defined in the previous section. We define the inclusion material density $\rho_{e}$ in each element $e, e=1,2, \ldots, N_{e}$ such that $\rho_{e}=1$ is associated with the inclusion/void phase and $\rho=0$ is associated with the matrix phase. The topology optimization is formulated as follows: 


$$
\begin{aligned}
\text { Maximize : } & \left|\bar{F}_{i j k l}(\boldsymbol{\rho})\right| \\
\text { subject : } & \mathbf{K U}=\mathbf{F} \\
: & \sum_{e=1}^{N_{e}} \rho_{e} v_{e} /\left(\sum_{e=1}^{N_{e}} v_{e}\right)=f \\
& 0 \leq \rho_{e} \leq 1, \quad e=1,2, \ldots, N_{e}
\end{aligned}
$$

The discrete system $\mathbf{K U}=\mathbf{F}$ is defined in Eqs. 23, -28 . Above, $v_{e}$ is the volume of an element $e$ and $f$ is the inclusion volume fraction.

We use the SIMP method [71, 72, 73] to solve the problem. In this framework, the local material properties are interpolated with respect to the local density in a continuous manner, using penalty exponents to enforce local densities to converge to values close to 0 or 1 . Selection of exponents have been investigated in [74]. For composites made of two phases, we use the following expression:

$$
\begin{aligned}
& {\left[C_{i j k l}(\rho)\right]=\rho^{p c}\left[C_{i j k l}^{1}\right]+\left(1-\rho^{p c}\right)\left[C_{i j k l}^{2}\right]} \\
& {\left[\alpha_{i j}(\rho)\right]=\rho^{p a}\left[\alpha_{i j}^{1}\right]+\left(1-\rho^{p a}\right)\left[\alpha_{i j}^{2}\right]} \\
& {\left[\mathscr{E}_{k i j}(\rho)\right]=\rho^{p e}\left[\mathscr{E}_{k i j}^{1}\right]+\left(1-\rho^{p e}\right)\left[\mathscr{E}_{k i j}^{2}\right]}
\end{aligned}
$$

where the superscript 1 and 2 are associated with phase 1 and phase 2 , respectively and $p c, p a$ and $p e$ are penalty exponents. In most of the numerical examples, these values are chosen as $p c=p a=p e=3$, except in a few cases where $p e=4$ is sometimes used to improve the convergence.

In the special case of porous materials, the following expression is usually preferred [73]:

$$
\begin{aligned}
& C_{i j k l}(\rho)=C_{i j k l}^{v o i d}+\rho^{p c} \times C_{i j k l}^{m} \\
& \alpha_{i j}(\rho)=\alpha_{i j}^{v o i d}+\rho^{p a} \times \alpha_{i j}^{m} \\
& \mathscr{E}_{k i j}(\rho)=\mathscr{E}_{k i j}^{v o i d}+\rho^{p e} \times \mathscr{E}_{k i j}^{m}
\end{aligned}
$$

where void refers to a fictitious material with small values of the (stiffness, dielectric, piezoelectric) properties to mimic the void. 
The above problem (40) requires evaluating the gradient of the objective function with respect to the local densities (subsequently referred to as sensitivities). The objective function, i.e. the effective flexoelectric tensor, is written in matrix form as (see Appendix A):

$$
[\overline{\mathbb{F}}(\rho)]=\left\langle\left(\mathbf{B}^{0}\right)^{T}: \mathbb{C}: \tilde{\mathbf{A}}^{1}-\left(\mathbf{h}^{0}\right)^{T} \cdot \mathscr{E}: \tilde{\mathbf{A}}^{1}-\left(\mathbf{B}^{0}\right)^{T}: \mathscr{E}^{T} \cdot \tilde{\mathbf{D}}^{1}-\left(\mathbf{h}^{0}\right)^{T} \cdot \boldsymbol{\alpha} \cdot \tilde{\mathbf{D}}^{1}\right\rangle
$$

where we have omitted the dependence to the coordinates $\mathbf{x}$ and design variable $\rho$ to alleviate the notations. The developed expressions for the sensitivities are given the next section. The optimization problem (40) is solved by the Conservative Convex Separable Appro ximations (CCSA optimizer[75].

\subsection{Numerical Analysis of Sensitivity}

The gradient of flexoelectric tensor $[\overline{\mathbb{F}}]$ with respect to $\rho$, is expressed as:

$$
\begin{aligned}
\frac{\partial[\overline{\mathbb{F}}]}{\partial \rho}= & \frac{\partial\left(\left(\mathbf{B}^{0}\right)^{T}[\mathbb{C}(\rho)] \tilde{\mathbf{A}}^{1}\right)}{\partial \rho}-\frac{\partial\left(\left(\mathbf{h}^{0}\right)^{T}[\mathscr{E}(\rho)] \tilde{\mathbf{A}}^{1}\right)}{\partial \rho}-\frac{\partial\left(\left(\mathbf{B}^{0}\right)^{T}[\mathscr{E}(\rho)]^{T} \tilde{\mathbf{D}}^{1}\right)}{\partial \rho}-\frac{\partial\left(\left(\mathbf{h}^{0}\right)^{T}[\boldsymbol{\alpha}(\rho)] \tilde{\mathbf{D}}^{1}\right)}{\partial \rho} \\
= & \frac{\partial\left\{\left(\mathbf{V}_{u}^{T}\right) \mathbf{B}_{u}^{T}[\mathbb{C}(\rho)] \mathbf{B}_{u}\left(\mathbf{W}_{u}-\mathbf{W}_{u}^{x}\right)\right\}}{\partial \rho}+\frac{\partial\left\{\left(\mathbf{V}_{\phi}^{T}\right) \mathbf{B}_{\phi}^{T}[\mathscr{E}(\rho)] \mathbf{B}_{u}\left(\mathbf{W}_{u}-\mathbf{W}_{u}{ }^{x}\right)\right\}}{\partial \rho} \\
& +\frac{\partial\left\{\left(\mathbf{V}_{u}^{T}\right) \mathbf{B}_{u}^{T}[\mathscr{E}(\rho)]^{T} \mathbf{B}_{\phi}\left(\mathbf{W}_{\phi}-\mathbf{W}_{\phi}^{x}\right)\right\}}{\partial \rho}-\frac{\partial\left\{\left(\mathbf{V}_{\phi}^{T}\right) \mathbf{B}_{\phi}^{T}[\boldsymbol{\alpha}(\rho)] \mathbf{B}_{\phi}\left(\mathbf{W}_{\phi}-\mathbf{W}_{\phi}{ }^{x}\right)\right\}}{\partial \rho}
\end{aligned}
$$


Expanding Eq. (44), we have

$$
\begin{aligned}
\frac{\partial[\overline{\mathbb{F}}]}{\partial \rho}= & \left\{\frac{\partial\left(\mathbf{V}_{u}^{T}\right)}{\partial \rho} \mathbf{B}_{u}^{T}[\mathbb{C}(\rho)] \mathbf{B}_{u}\left(\mathbf{W}_{u}-\mathbf{W}_{u}^{x}\right)+\left(\mathbf{V}_{u}\right)^{T} \frac{\partial\left(\mathbf{B}_{u}^{T}[\mathbb{C}(\rho)] \mathbf{B}_{u}\right)}{\partial \rho}\left(\mathbf{W}_{u}-\mathbf{W}_{u}{ }^{x}\right)\right. \\
& \left.+\left(\mathbf{V}_{u}^{T}\right) \mathbf{B}_{u}^{T}[\mathbb{C}(\rho)] \mathbf{B}_{u} \frac{\partial\left(\mathbf{W}_{u}-\mathbf{W}_{u}{ }^{x}\right)}{\partial \rho}\right\}+\left\{\frac{\partial\left(\mathbf{V}_{\phi}^{T}\right)}{\partial \rho} \mathbf{B}_{\phi}^{T}[\mathscr{E}(\rho)] \mathbf{B}_{u}\left(\mathbf{W}_{u}-\mathbf{W}_{u}^{x}\right)\right. \\
& \left.+\left(\mathbf{V}_{\phi}\right)^{T} \frac{\partial\left(\mathbf{B}_{\phi}^{T}[\mathscr{E}(\rho)] \mathbf{B}_{u}\right)}{\partial \rho}\left(\mathbf{W}_{u}-\mathbf{W}_{u}^{x}\right)+\left(\mathbf{V}_{\phi}^{T}\right) \mathbf{B}_{\phi}^{T}[\mathscr{E}(\rho)] \mathbf{B}_{u} \frac{\partial\left(\mathbf{W}_{u}-\mathbf{W}_{u}{ }^{x}\right)}{\partial \rho}\right\} \\
& +\left\{\frac{\partial\left(\mathbf{V}_{u}^{T}\right)}{\partial \rho} \mathbf{B}_{u}^{T}[\mathscr{E}(\rho)]^{T} \mathbf{B}_{\phi}\left(W_{\phi}-\mathbf{W}_{\phi}^{x}\right)+\left(\mathbf{V}_{u}\right)^{T} \frac{\partial\left(\mathbf{B}_{u}^{T}[\mathscr{E}(\rho)]^{T} \mathbf{B}_{\phi}\right)}{\partial \rho}\left(\mathbf{W}_{\phi}-\mathbf{W}_{\phi}{ }^{x}\right)\right. \\
& \left.+\left(\mathbf{V}_{u}^{T}\right) \mathbf{B}_{u}^{T}[\mathscr{E}(\rho)]^{T} \mathbf{B}_{\phi} \frac{\partial\left(\mathbf{W}_{\phi}-\mathbf{W}_{\phi}^{x}\right)}{\partial \rho}\right\}-\left\{\frac{\partial\left(\mathbf{V}_{\phi}^{T}\right)}{\partial \rho} \mathbf{B}_{\phi}^{T}[\boldsymbol{\alpha}(\rho)] \mathbf{B}_{\phi}\left(\mathbf{W}_{\phi}-\mathbf{W}_{\phi}{ }^{x}\right)\right. \\
& \left.+\left(\mathbf{V}_{\phi}\right)^{T} \frac{\partial\left(\mathbf{B}_{\phi}^{T}[\boldsymbol{\alpha}(\rho)] \mathbf{B}_{\phi}\right)}{\partial \rho}\left(\mathbf{W}_{\phi}-\mathbf{W}_{\phi}^{x}\right)+\left(\mathbf{V}_{\phi}^{T}\right) \mathbf{B}_{\phi}^{T}[\boldsymbol{\alpha}(\rho)] \mathbf{B}_{\phi} \frac{\partial\left(\mathbf{W}_{\phi}-\mathbf{W}_{\phi}^{x}\right)}{\partial \rho}\right\}
\end{aligned}
$$

After rearranging Eq. (45), we obtain

$$
\begin{aligned}
\frac{\partial[\overline{\mathbb{F}}]}{\partial \rho}= & \frac{\partial\left(\mathbf{V}_{u}^{T}\right)}{\partial \rho}\left\{\mathbf{B}_{u}^{T}[\mathbb{C}(\rho)] \mathbf{B}_{u}\left(\mathbf{W}_{u}-\mathbf{W}_{u}{ }^{x}\right)+\mathbf{B}_{u}^{T}[\mathscr{E}(\rho)]^{T} \mathbf{B}_{\phi}\left(W_{\phi}-\mathbf{W}_{\phi}{ }^{x}\right)\right\} \\
& +\frac{\partial\left(\mathbf{V}_{\phi}^{T}\right)}{\partial \rho}\left\{\mathbf{B}_{\phi}^{T}[\mathscr{E}(\rho)] \mathbf{B}_{u}\left(\mathbf{W}_{u}-\mathbf{W}_{u}{ }^{x}\right)-\mathbf{B}_{\phi}^{T}[\boldsymbol{\alpha}(\rho)] \mathbf{B}_{\phi}\left(\mathbf{W}_{\phi}-\mathbf{W}_{\phi}{ }^{x}\right)\right\} \\
& +\left\{\left(\mathbf{V}_{u}^{T}\right) \mathbf{B}_{u}^{T}[\mathbb{C}(\rho)] \mathbf{B}_{u}+\left(\mathbf{V}_{\phi}{ }^{T}\right) \mathbf{B}_{\phi}^{T}[\mathscr{E}(\rho)] \mathbf{B}_{u}\right\} \frac{\partial\left(\mathbf{W}_{u}-\mathbf{W}_{u}{ }^{x}\right)}{\partial \rho} \\
& +\left\{\left(\mathbf{V}_{u}^{T}\right) \mathbf{B}_{u}^{T}[\mathscr{E}(\rho)]^{T} \mathbf{B}_{\phi}-\left(\mathbf{V}_{\phi}{ }^{T}\right) \mathbf{B}_{\phi}^{T}[\boldsymbol{\alpha}(\rho)] \mathbf{B}_{\phi}\right\} \frac{\partial\left(\mathbf{W}_{\phi}-\mathbf{W}_{\phi}{ }^{x}\right)}{\partial \rho} \\
& +\left(\mathbf{V}_{u}\right)^{T} \frac{\partial\left(\mathbf{B}_{u}^{T}[\mathbb{C}(\rho)] \mathbf{B}_{u}\right)}{\partial x}\left(\mathbf{W}_{u}-\mathbf{W}_{u}{ }^{x}\right)+\left(\mathbf{V}_{\phi}\right)^{T} \frac{\partial\left(\mathbf{B}_{\phi}^{T}[\mathscr{E}(\rho)] \mathbf{B}_{u}\right)}{\partial x}\left(\mathbf{W}_{u}-\mathbf{W}_{u}{ }^{x}\right) \\
& +\left(\mathbf{V}_{u}\right)^{T} \frac{\partial\left(\mathbf{B}_{u}^{T}[\mathscr{E}(\rho)]^{T} \mathbf{B}_{\phi}\right)}{\partial \rho}\left(\mathbf{W}_{\phi}-\mathbf{W}_{\phi}{ }^{x}\right)-\left(\mathbf{V}_{\phi}\right)^{T} \frac{\partial\left(\mathbf{B}_{\phi}^{T}[\boldsymbol{\alpha}(\rho)] \mathbf{B}_{\phi}\right)}{\partial \rho}\left(\mathbf{W}_{\phi}-\mathbf{W}_{\phi}{ }^{x}\right)
\end{aligned}
$$

The adjoint method has been widely used for sensitivity analysis of gradient-based optimization algorithms [76, 77], and is employed here. The corresponding Lagrangian for the optimization problem (40) is formed by introducing an adjoint vector $\lambda$ as:

$$
L=\overline{\mathbb{F}}+\lambda(\mathbf{K U}-\mathbf{F})
$$


As $\mathbf{K U}-\mathbf{F}=\mathbf{0}$ holds, then $\lambda=\left[\lambda_{i}, \mu_{i}\right](i=1,2,3)$ can take arbitrary values. Differentiating the Lagrangian $L$ with respect to the design variable $\rho$ gives:

$$
\frac{\partial L}{\partial \rho}=\frac{\partial \overline{\mathbb{F}}}{\partial \rho}+\lambda \frac{\partial(\mathbf{K} \mathbf{U}-\mathbf{F})}{\partial \rho}
$$

The vector $\mathbf{K U}=\mathbf{F}$ is defined in Eqs. 23)-28. Substituting these equations into (48), then splitting the expression (46) into 4 parts, denoted by $I, I I, I I I, I V$, we have:

$$
\begin{aligned}
\frac{\partial\left[\overline{\mathbb{F}}^{I}\right]}{\partial \rho}= & \frac{\partial\left(\mathbf{V}_{u}^{T}\right)}{\partial \rho}\left\{\mathbf{B}_{u}^{T}[\mathbb{C}(\rho)] \mathbf{B}_{u}\left(\mathbf{W}_{u}-\mathbf{W}_{u}{ }^{x}\right)+\mathbf{B}_{u}^{T}[\mathscr{E}(\rho)]^{T} \mathbf{B}_{\phi}\left(W_{\phi}-\mathbf{W}_{\phi}{ }^{x}\right)\right\} \\
& +\frac{\partial\left(\mathbf{V}_{\phi}^{T}\right)}{\partial \rho}\left\{\mathbf{B}_{\phi}^{T}[\mathscr{E}(\rho)] \mathbf{B}_{u}\left(\mathbf{W}_{u}-\mathbf{W}_{u}{ }^{x}\right)-\mathbf{B}_{\phi}^{T}[\boldsymbol{\alpha}(\rho)] \mathbf{B}_{\phi}\left(\mathbf{W}_{\phi}-\mathbf{W}_{\phi}{ }^{x}\right)\right\} \\
& +\left\{\frac{\partial\left(\mathbf{V}_{\phi}^{T}\right)}{\partial \rho} \mathbf{K}_{\phi \phi}+\left(\mathbf{V}_{\phi}^{T}\right) \frac{\partial \mathbf{K}_{\phi \phi}}{\partial \rho}+\frac{\partial\left(\mathbf{V}_{u}^{T}\right)}{\partial \rho} \mathbf{K}_{\phi u}^{T}+\left(\mathbf{V}_{u}^{T}\right) \frac{\partial \mathbf{K}_{\phi u}^{T}}{\partial \rho}\right\} \boldsymbol{\lambda}_{1} \\
& +\left\{-\frac{\partial\left(\mathbf{V}_{\phi}^{T}\right)}{\partial \rho} \mathbf{K}_{\phi u}-\left(\mathbf{V}_{\phi}^{T}\right) \frac{\partial \mathbf{K}_{\phi u}}{\partial \rho}+\frac{\partial\left(\mathbf{V}_{u}^{T}\right)}{\partial \rho} \mathbf{K}_{u u}+\left(\mathbf{V}_{u}^{T}\right) \frac{\partial \mathbf{K}_{u u}}{\partial \rho}\right\} \boldsymbol{\mu}_{1} \\
= & \frac{\partial\left(\mathbf{V}_{u}^{T}\right)}{\partial \rho}\left\{\mathbf{B}_{u}^{T}[\mathbb{C}(\rho)] \mathbf{B}_{u}\left(\mathbf{W}_{u}-\mathbf{W}_{u}{ }^{x}\right)+\mathbf{B}_{u}^{T}[\mathscr{E}(\rho)]^{T} \mathbf{B}_{\phi}\left(W_{\phi}-\mathbf{W}_{\phi}{ }^{x}\right)+\mathbf{K}_{\phi u}^{T} \boldsymbol{\lambda}_{1}+\mathbf{K}_{u u} \boldsymbol{\mu}_{1}\right\} \\
& +\frac{\partial\left(\mathbf{V}_{\phi}^{T}\right)}{\partial \rho}\left\{\mathbf{B}_{\phi}^{T}[\mathscr{E}(\rho)] \mathbf{B}_{u}\left(\mathbf{W}_{u}-\mathbf{W}_{u}{ }^{x}\right)-\mathbf{B}_{\phi}^{T}[\boldsymbol{\alpha}(\rho)] \mathbf{B}_{\phi}\left(\mathbf{W}_{\phi}-\mathbf{W}_{\phi}{ }^{x}\right)+\mathbf{K}_{\phi \phi} \boldsymbol{\lambda}_{1}-\mathbf{K}_{\phi u} \boldsymbol{\mu}_{1}\right\} \\
& +\left\{\left(\mathbf{V}_{\phi}^{T}\right) \frac{\partial \mathbf{K}_{\phi \phi}}{\partial \rho}+\left(\mathbf{V}_{u}^{T}\right) \frac{\partial \mathbf{K}_{\phi u}^{T}}{\partial \rho}\right\} \boldsymbol{\lambda}_{1}+\left\{-\left(\mathbf{V}_{\phi}^{T}\right) \frac{\partial \mathbf{K}_{\phi u}}{\partial \rho}+\left(\mathbf{V}_{u}^{T}\right) \frac{\left.\left.\partial \mathbf{K}_{u u}\right)\right\} \boldsymbol{\mu}_{1}}{\partial \rho}\right.
\end{aligned}
$$

As Eq. (49) holds for arbitrary vectors $\boldsymbol{\lambda}_{1}$ and $\boldsymbol{\mu}_{1}$, the adjoint vectors $\boldsymbol{\lambda}_{1}$ and $\boldsymbol{\mu}_{1}$ can be chosen as the solution of the following adjoint equation to eliminate the implicit terms $\frac{\partial\left(\mathbf{V}_{u}^{T}\right)}{\partial \rho}$ and $\frac{\partial\left(\mathbf{V}_{\phi}^{T}\right)}{\partial \rho}$. Then the corresponding adjoint problem is defined as,

$$
\begin{array}{r}
\frac{\partial\left(\mathbf{V}_{u}^{T}\right)}{\partial \rho}\left\{\mathbf{B}_{u}^{T}[\mathbb{C}(\rho)] \mathbf{B}_{u}\left(\mathbf{W}_{u}-\mathbf{W}_{u}{ }^{x}\right)+\mathbf{B}_{u}^{T}[\mathscr{E}(\rho)]^{T} \mathbf{B}_{\phi}\left(W_{\phi}-\mathbf{W}_{\phi}{ }^{x}\right)+\mathbf{K}_{\phi u}^{T} \boldsymbol{\lambda}_{1}+\mathbf{K}_{u u} \boldsymbol{\mu}_{1}\right\}=0 \\
\frac{\partial\left(\mathbf{V}_{\phi}{ }^{T}\right)}{\partial \rho}\left\{\mathbf{B}_{\phi}^{T}[\mathscr{E}(\rho)] \mathbf{B}_{u}\left(\mathbf{W}_{u}-\mathbf{W}_{u}{ }^{x}\right)-\mathbf{B}_{\phi}^{T}[\boldsymbol{\alpha}(\rho)] \mathbf{B}_{\phi}\left(\mathbf{W}_{\phi}-\mathbf{W}_{\phi}{ }^{x}\right)+\mathbf{K}_{\phi \phi} \boldsymbol{\lambda}_{1}-\mathbf{K}_{\phi u} \boldsymbol{\mu}_{1}\right\}=0
\end{array}
$$


and written in matrix form, gives

$$
\left[\begin{array}{cc}
\mathbf{K}_{\phi \phi} & -\mathbf{K}_{\phi u} \\
\mathbf{K}_{\phi u}^{T} & \mathbf{K}_{u u}
\end{array}\right]\left[\begin{array}{c}
\lambda_{1} \\
\boldsymbol{\mu}_{1}
\end{array}\right]=-\left[\begin{array}{c}
\mathbf{B}_{\phi}^{T}[\mathscr{E}(\rho)] \mathbf{B}_{u}\left(\mathbf{W}_{u}-\mathbf{W}_{u}{ }^{x}\right)-\mathbf{B}_{\phi}^{T}[\alpha(\rho)] \mathbf{B}_{\phi}\left(\mathbf{W}_{\phi}-\mathbf{W}_{\phi}{ }^{x}\right) \\
\mathbf{B}_{u}^{T}[\mathbb{C}(\rho)] \mathbf{B}_{u}\left(\mathbf{W}_{u}-\mathbf{W}_{u}{ }^{x}\right)+\mathbf{B}_{u}^{T}[\xi(\rho)]^{T} \mathbf{B}_{\phi}\left(W_{\phi}-\mathbf{W}_{\phi}{ }^{x}\right)
\end{array}\right]
$$

Part $I I$ of 49 ) is given by

$$
\begin{aligned}
\frac{\partial\left[\overline{\mathbb{F}}^{I I}\right]}{\partial \rho}= & \left\{\left(\mathbf{V}_{u}^{T}\right) \mathbf{B}_{u}^{T}[\mathbb{C}(\rho)] \mathbf{B}_{u}+\left(\mathbf{V}_{\phi}^{T}\right) \mathbf{B}_{\phi}^{T}[\mathscr{E}(\rho)] \mathbf{B}_{u}\right\} \frac{\partial \mathbf{W}_{u}}{\partial \rho} \\
& +\left\{\left(\mathbf{V}_{u}^{T}\right) \mathbf{B}_{u}^{T}[\mathscr{E}(\rho)]^{T} \mathbf{B}_{\phi}-\left(\mathbf{V}_{\phi}^{T}\right) \mathbf{B}_{\phi}^{T}[\boldsymbol{\alpha}(\rho)] \mathbf{B}_{\phi}\right\} \frac{\partial \mathbf{W}_{\phi}}{\partial \rho} \\
& +\boldsymbol{\lambda}_{2}^{T}\left\{\frac{\partial \mathbf{K}_{\phi \phi}}{\partial \rho} \mathbf{W}_{\phi}+\mathbf{K}_{\phi \phi} \frac{\partial \mathbf{W}_{\phi}}{\partial \rho}+\frac{\partial \mathbf{K}_{\phi u}}{\partial \rho} \mathbf{W}_{u}+\mathbf{K}_{\phi u} \frac{\partial \mathbf{W}_{u}}{\partial \rho}-\frac{\partial \mathbf{F}_{\phi}}{\partial \rho}\right\} \\
& +\boldsymbol{\mu}_{2}^{T}\left\{-\frac{\partial \mathbf{K}_{\phi u}^{T}}{\partial \rho} \mathbf{W}_{\phi}-\mathbf{K}_{\phi u}^{T} \frac{\partial \mathbf{W}_{\phi}}{\partial \rho}+\frac{\partial \mathbf{K}_{u u}}{\partial \rho} \mathbf{W}_{u}+\mathbf{K}_{u u} \frac{\partial \mathbf{W}_{u}}{\partial \rho}-\frac{\partial \mathbf{F}_{u}}{\partial \rho}\right\} \\
= & \left\{\mathbf{V}_{u}^{T} \mathbf{B}_{u}^{T}[\mathbb{C}(\rho)] \mathbf{B}_{u}+\mathbf{V}_{\phi}^{T} \mathbf{B}_{\phi}^{T}[\mathscr{E}(\rho)] \mathbf{B}_{u}+\boldsymbol{\lambda}_{2}^{T} \mathbf{K}_{\phi u}+\boldsymbol{\mu}_{2}^{T} \mathbf{K}_{u u}\right\} \frac{\partial \mathbf{W}_{u}}{\partial \rho} \\
& +\left\{\left(\mathbf{V}_{u}^{T}\right) \mathbf{B}_{u}^{T}[\mathscr{E}(\rho)]^{T} \mathbf{B}_{\phi}-\left(\mathbf{V}_{\phi}^{T}\right) \mathbf{B}_{\phi}^{T}[\boldsymbol{\alpha}(\rho)] \mathbf{B}_{\phi}+\boldsymbol{\lambda}_{2}^{T} \mathbf{K}_{\phi, \phi}-\boldsymbol{\mu}_{2}^{T} \mathbf{K}_{\phi u}^{T}\right\} \frac{\partial \mathbf{W}_{\phi}}{\partial \rho} \\
& +\boldsymbol{\lambda}_{2}^{T}\left\{\frac{\partial \mathbf{K}_{\phi \phi}}{\partial \rho} \mathbf{W}_{\phi}+\frac{\partial \mathbf{K}_{\phi u}}{\partial \rho} \mathbf{W}_{u}-\frac{\partial \mathbf{F}_{\phi}}{\partial \rho}\right\}+\boldsymbol{\mu}_{2}^{T}\left\{-\frac{\partial \mathbf{K}_{\phi u}^{T}}{\partial \rho} \mathbf{W}_{\phi}+\frac{\partial \mathbf{K}_{u u}}{\partial \rho} \mathbf{W}_{u}-\frac{\partial \mathbf{F}_{u}}{\partial \rho}\right\}
\end{aligned}
$$

The derivatives of body forces with respect to $\rho$ must also be evaluated. Using the same method as for Part $I$, after eliminating the displacement derivatives, the adjoint problem for Eq. [52] reads:

$$
\left[\begin{array}{cc}
\mathbf{K}_{\phi \phi} & -\mathbf{K}_{\phi u} \\
\mathbf{K}_{\phi u}^{T} & \mathbf{K}_{u u}
\end{array}\right]\left[\begin{array}{c}
\lambda_{2} \\
\boldsymbol{\mu}_{2}
\end{array}\right]=-\left[\begin{array}{c}
\mathbf{B}_{\phi}^{T}[\mathscr{E}(\rho)] \mathbf{B}_{u} \mathbf{V}_{u}-\mathbf{B}_{\phi}^{T}[\boldsymbol{\alpha}(\rho)] \mathbf{B}_{\phi} \mathbf{V}_{\phi} \\
\mathbf{B}_{u}^{T}[\mathbb{C}(\rho)] \mathbf{B}_{u} \mathbf{V}_{u}+\mathbf{B}_{u}^{T}[\mathscr{E}(\rho)]^{T} \mathbf{B}_{\phi} \mathbf{V}_{\phi}
\end{array}\right]
$$


Similarly:

$$
\begin{aligned}
\frac{\partial\left[\overline{\mathbb{F}}^{I I I}\right]}{\partial \rho}= & \left\{\left(\mathbf{V}_{u}^{T}\right) \mathbf{B}_{u}^{T}[\mathbb{C}(\rho)] \mathbf{B}_{u}+\left(\mathbf{V}_{\phi}^{T}\right) \mathbf{B}_{\phi}^{T}[\mathscr{E}(\rho)] \mathbf{B}_{u}\right\}\left(-\frac{\partial W_{u}^{x}}{\partial \rho}\right) \\
& +\left\{\left(\mathbf{V}_{u}^{T}\right) \mathbf{B}_{u}^{T}[\mathscr{E}(\rho)]^{T} \mathbf{B}_{\phi}-\left(\mathbf{V}_{\phi}^{T}\right) \mathbf{B}_{\phi}^{T}[\boldsymbol{\alpha}(\rho)] \mathbf{B}_{\phi}\right\}\left(-\frac{\partial \mathbf{W}_{\phi}{ }^{x}}{\partial \rho}\right) \\
& +\boldsymbol{\lambda}_{3}^{T}\left\{\frac{\partial \mathbf{K}_{\phi \phi}}{\partial \rho} U_{\phi}+\mathbf{K}_{\phi \phi} \frac{\partial U_{\phi}}{\partial \rho}+\frac{\partial \mathbf{K}_{\phi u}}{\partial \rho} U_{u}+\mathbf{K}_{\phi u} \frac{\partial U_{u}}{\partial \rho}\right\} \\
& +\boldsymbol{\mu}_{3}^{T}\left\{-\frac{\partial \mathbf{K}_{\phi u}^{T}}{\partial \rho} U_{\phi}-\mathbf{K}_{\phi u}^{T} \frac{\partial U_{\phi}}{\partial \rho}+\frac{\partial \mathbf{K}_{u u}}{\partial \rho} U_{u}+\mathbf{K}_{u u} \frac{\partial U_{u}}{\partial \rho}\right\} \\
= & \left\{-\mathbf{V}_{u}^{T} \mathbf{B}_{u}^{T}[\mathbb{C}(\rho)] \mathbf{B}_{u} x-\mathbf{V}_{\phi}^{T} \mathbf{B}_{\phi}^{T}[\mathscr{E}(\rho)] \mathbf{B}_{u} x+\lambda_{3}^{T} \mathbf{K}_{\phi u}+\boldsymbol{\mu}_{3} \mathbf{K}_{u u}\right\} \frac{\partial U_{u}}{\partial \rho} \\
& +\left\{-\mathbf{V}_{u}^{T} \mathbf{B}^{T}[\mathscr{E}(\rho)]^{T} \mathbf{B}_{\phi} x+\left(\mathbf{V}_{\phi}^{T}\right) \mathbf{B}_{\phi}^{T}[\boldsymbol{\alpha}(\rho)] \mathbf{B}_{\phi} x+\lambda_{3}^{T} \mathbf{K}_{\phi \phi}-\mu_{3}^{T} \mathbf{K}_{\phi u}^{T}\right\} \frac{\partial U_{\phi}}{\partial \rho} \\
& +\lambda_{3}^{T}\left\{\frac{\partial \mathbf{K}_{\phi \phi}}{\partial \rho} U_{\phi}+\frac{\partial \mathbf{K}_{\phi u}}{\partial \rho} U_{u}\right\}+\boldsymbol{\mu}_{3}^{T}\left\{-\frac{\partial \mathbf{K}_{\phi u}^{T}}{\partial \rho} U_{\phi}+\frac{\partial \mathbf{K}_{u u}}{\partial \rho} U_{u}\right\}
\end{aligned}
$$

The adjoint problem for Part $I I I$ is written as:

$$
\left[\begin{array}{cc}
\mathbf{K}_{\phi \phi} & -\mathbf{K}_{\phi u} \\
\mathbf{K}_{\phi u}^{T} & \mathbf{K}_{u u}
\end{array}\right]\left[\begin{array}{c}
\lambda_{3} \\
\boldsymbol{\mu}_{3}
\end{array}\right]=-\left[\begin{array}{c}
-\mathbf{B}_{\phi}^{T}[\mathscr{E}(\rho)] \mathbf{B}_{u} \mathbf{V}_{u} \mathbf{x}+\mathbf{B}_{\phi}^{T}[\alpha(\rho)] \mathbf{B}_{\phi} \mathbf{V}_{\phi} \mathbf{x} \\
-\mathbf{B}_{u}^{T}[\mathbb{C}(\rho)] \mathbf{B}_{u} \mathbf{V}_{u} \mathbf{x}-\mathbf{B}_{u}^{T}[\mathscr{E}(\rho)]^{T} \mathbf{B}_{\phi} \mathbf{V}_{\phi} \mathbf{x}
\end{array}\right]
$$

From Eq. 46, we can obtain Part $I V$, which is explicit and can be easily calculated in terms the interpolation function Eq.441].

$$
\begin{aligned}
\frac{\partial\left[\overline{\mathbb{F}}^{I V}\right]}{\partial \rho}= & \left(\mathbf{V}_{u}\right)^{T} \mathbf{B}_{u}^{T} \frac{\partial([\mathbb{C}(\rho)])}{\partial x} \mathbf{B}_{u}\left(\mathbf{W}_{u}-\mathbf{W}_{u}{ }^{x}\right)+\left(\mathbf{V}_{\phi}\right)^{T} \mathbf{B}_{\phi}^{T} \frac{\partial([\mathscr{E}(\rho)])}{\partial x} \mathbf{B}_{u}\left(\mathbf{W}_{u}-\mathbf{W}_{u}{ }^{x}\right) \\
& +\left(\mathbf{V}_{u}\right)^{T} \mathbf{B}_{u}^{T} \frac{\partial([\mathscr{E}(\rho)])}{\partial \rho} \mathbf{B}_{\phi}\left(\mathbf{W}_{\phi}-\mathbf{W}_{\phi}{ }^{x}\right)-\left(\mathbf{V}_{\phi}\right)^{T} \mathbf{B}_{\phi}^{T} \frac{\partial([\boldsymbol{\alpha}(\rho)])}{\partial \rho} \mathbf{B}_{\phi}\left(\mathbf{W}_{\phi}-\mathbf{W}_{\phi}{ }^{x}\right)
\end{aligned}
$$

After solving all the above adjoint problems, we can get the whole explicit sensi- 
tivity of flexoelectric tensor with respect to density $\rho$ as:

$$
\begin{aligned}
\frac{\partial[\overline{\mathbb{F}}]}{\partial \rho}= & \left\{\left(\mathbf{V}_{\phi}^{T}\right) \frac{\partial \mathbf{K}_{\phi \phi}}{\partial \rho}+\left(\mathbf{V}_{u}^{T}\right) \frac{\partial \mathbf{K}_{\phi u}^{T}}{\partial \rho}\right\} \boldsymbol{\lambda}_{1}+\left\{-\left(\mathbf{V}_{\phi}^{T}\right) \frac{\partial \mathbf{K}_{\phi u}}{\partial \rho}+\left(\mathbf{V}_{u}^{T}\right) \frac{\partial \mathbf{K}_{u u}}{\partial \rho}\right\} \boldsymbol{\mu}_{1} \\
& +\boldsymbol{\lambda}_{2}^{T}\left\{\frac{\partial \mathbf{K}_{\phi \phi}}{\partial \rho} \mathbf{W}_{\phi}+\frac{\partial \mathbf{K}_{\phi u}}{\partial \rho} \mathbf{W}_{u}-\frac{\partial \mathbf{F}_{\phi}}{\partial \rho}\right\}+\boldsymbol{\mu}_{2}^{T}\left\{-\frac{\partial \mathbf{K}_{\phi u}^{T}}{\partial \rho} \mathbf{W}_{\phi}+\frac{\partial \mathbf{K}_{u u}}{\partial \rho} \mathbf{W}_{u}-\frac{\partial \mathbf{F}_{u}}{\partial \rho}\right\} \\
& +\boldsymbol{\lambda}_{3}^{T}\left\{\frac{\partial \mathbf{K}_{\phi \phi}}{\partial \rho} \mathbf{W}_{\phi}^{x}+\frac{\partial \mathbf{K}_{\phi u}}{\partial \rho} \mathbf{W}_{u}{ }^{x}\right\}+\boldsymbol{\mu}_{3}^{T}\left\{-\frac{\partial \mathbf{K}_{\phi u}^{T}}{\partial \rho} \mathbf{W}_{\phi}^{x}+\frac{\partial \mathbf{K}_{u u}}{\partial \rho} \mathbf{W}_{u}{ }^{x}\right\} \\
& +\left(\mathbf{V}_{u}\right)^{T} \frac{\partial\left(\mathbf{B}_{u}^{T}[\mathbb{C}(\rho)] \mathbf{B}_{u}\right)}{\partial \rho}\left(\mathbf{W}_{u}-\mathbf{W}_{u}{ }^{x}\right)+\left(\mathbf{V}_{\phi}\right)^{T} \frac{\partial\left(B_{\phi}^{T}[\mathscr{E}(\rho)] \mathbf{B}_{u}\right)}{\partial x}\left(\mathbf{W}_{u}-\mathbf{W}_{u}{ }^{x}\right) \\
& +\left(\mathbf{V}_{u}\right)^{T} \frac{\partial\left(\mathbf{B}_{u}^{T}[\xi(\rho)]^{T} \mathbf{B}_{\phi}\right)}{\partial \rho}\left(\mathbf{W}_{\phi}-\mathbf{W}_{\phi}^{x}\right)-\left(\mathbf{V}_{\phi}\right)^{T} \frac{\partial\left(\mathbf{B}_{\phi}^{T}[\alpha(\rho)] \mathbf{B}_{\phi}\right)}{\partial \rho}\left(\mathbf{W}_{\phi}-\mathbf{W}_{\phi}{ }^{x}\right)
\end{aligned}
$$

The body forces are non-zero for the third adjoint problem. The interested readers can also calculate the derivative of body forces with respect to design variables by the presented adjoint method. Here we obtain 3 adjoint problems to compute the adjoint vectors $\boldsymbol{\lambda}_{1}$ and $\boldsymbol{\mu}_{1}, \boldsymbol{\lambda}_{2}$ and $\boldsymbol{\mu}_{2}$, as well as $\boldsymbol{\lambda}_{3}$ and $\boldsymbol{\mu}_{3}$, respectively. So we must choose 3 boundary conditions for the adjoint problems. Usually, we can define the same boundary conditions as the corresponding to equivalent finite element equations. However the numerical simulations indicate that prescribing one or two adjoint problems as zero Dirichlet boundary conditions leads to better numerical stability and larger absolute value of optimized components.

\section{Numerical examples}

In this section, the proposed computational homogenization framework is applied to optimize the components of the effective flexoelectric tensor of a two-phase composite. More specifically, we investigate the optimization of the $\bar{F}_{1221}, \bar{F}_{2221}, \bar{F}_{1112}$ and $\bar{F}_{2112}$ coefficients, as these coefficients characterize polarization under the action of bending. The other coefficients of the flexoelectric tensor, i.e. $\bar{F}_{1111}, \bar{F}_{2111}, \bar{F}_{1222}, \bar{F}_{2222}$ are not investigated here, as they correspond to polarization under more complex strain 
gradient modes. The homogenization and optimization is performed on a periodic heterogeneous material composed of piezoelectric phases. We consider three cases: (1) a composite made of two stiff piezoelectric phases; (2) a composite made of a stiff piezoelectric matrix and soft polymer inclusion; (3) a porous piezoelectric material. In all numerical examples, the RVEs are discretized by $60 \times 608$-node quadratic finite elements.

\subsection{Ceramic/ceramic Piezoelectric composite}

We first consider a two-phase composite made of piezoelectric phases. Each phase is made with PZT (lead zirconium titanate ceramics). To induce a heterogeneity, the crystal lattice is oriented by a mismatch angle of $\theta=\pi$ in the inclusion phase. The related properties of the matrix and inclusion are indicated in matrix form in $(58)-(61)$, in which the subscript $m$ and $i$ refer to the matrix and inclusion, respectively[78].

$$
\begin{aligned}
& {\left[\mathbf{C}^{m}\right]=\left[\mathbf{C}^{i}\right]=\left[\begin{array}{ccc}
131.39 & 83.237 & 0 \\
83.237 & 154.837 & 0 \\
0 & 0 & 35.8
\end{array}\right](G P a,} \\
& {\left[\boldsymbol{\alpha}^{m}\right]=\left[\boldsymbol{\alpha}^{i}\right]=\left[\begin{array}{cc}
2.079 & 0 \\
0 & 4.065
\end{array}\right]\left(C \cdot m^{-2}\right)} \\
& {\left[\mathscr{E}^{m}\right]=\left[\begin{array}{ccc}
-2.120582 & -2.120582 & 0 \\
0 & 0 & 0
\end{array}\right]\left(n C \cdot m^{-1} \cdot V^{-1}\right)} \\
& {\left[\mathscr{E}^{\mathscr{i}}\right]=\left[\begin{array}{ccc}
2.120582 & 2.120582 & 0 \\
0 & 0 & 0
\end{array}\right]\left(n C \cdot m^{-1} \cdot V^{-1}\right)}
\end{aligned}
$$

We perform the topology optimization of the inclusion shape with respect to the flexoelectric coefficients $\bar{F}_{1221}$ and $\bar{F}_{2112}$, and set the inclusion volume fraction to $f=$ 0.4. As a first guess, the design variables are uniformly set to $\rho_{e}=0.4(e=1, \ldots, N e=$ 3600). The guess design with triangular shape which is illustrated in Fig. 2a has been 


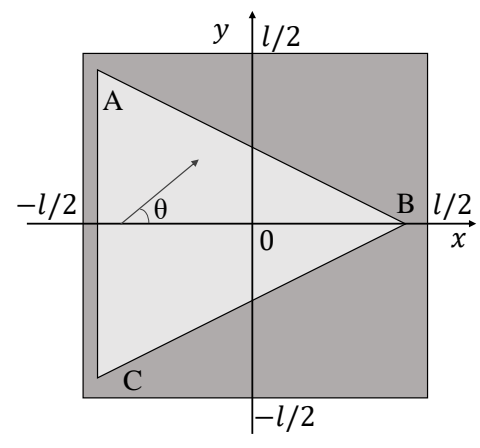

(a)

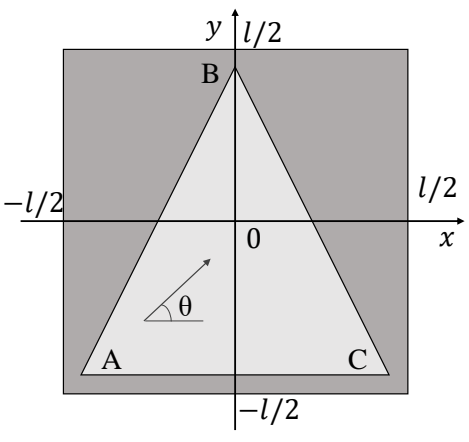

(b)

Figure 2: Unit cells with triangular inclusions with inclusion volume fraction of $f=0.4$, polarization $\mathrm{P}$ and the mismatch angle $\theta$ between matrix and inclusion phases; (a) guess design used for computing $\bar{F}_{1221}$ and $\bar{F}_{2112}$; (b) guess design used for computing $\bar{F}_{1112}$ and $\bar{F}_{2221}$.

investigated in [63] and will serve as a comparison solution with respect to optimized topological designs.

The final optimized unit cell topologies are shown in Figs. $4 \mathrm{a}$ and $7 \mathrm{a}$, where the optimization process converges in about 60 iterations for $\bar{F}_{1221}$ and $\bar{F}_{2112}$. In all figures, the copper and black colors refer to the inclusion and matrix phases, respectively. Iteration histories are shown in Fig. 3, where Normalized Flexoelectric F refers to $\bar{F}_{i j k l} / \bar{F}_{i j k l}^{R e f}$, where $\bar{F}_{i j k l}^{R e f}$ is the guess solution obtained on a unit cell with triangular shape inclusion.

The final values for the optimized microstructures are $\bar{F}_{1221}=1.365 \times 10^{-4}{\mathrm{C} . \mathrm{m}^{-1}}^{-1}$ and $\bar{F}_{2112}=2.689 \times 10^{-4} \mathrm{C} . \mathrm{m}^{-1}$, which represents a significant improvement of the values as compared to the reference triangular solutions of $79.61 \%$ and $83.55 \%$ for $\bar{F}_{1221}$ and $\bar{F}_{2112}$, respectively. It should be noticed that the obtained values are higher than naturally flexoelectric materials, such as $\mathrm{BaTiO}_{3}$ and PMN-PT[7] whose flexoelectricity is reported as of the order of $10^{-5} \mathrm{C} \cdot \mathrm{m}^{-1}$. It is worth noting that the present SIMP framework allows initiating the topology as uniform, with densities equal to the required volume fraction. This explains why the flexoelectric coefficients are initially zero.

In the next example, we analyze the size effects related to varying the length $\ell$ of 


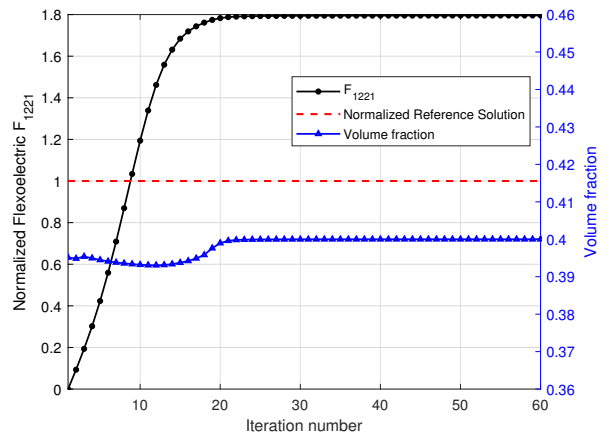

(a)

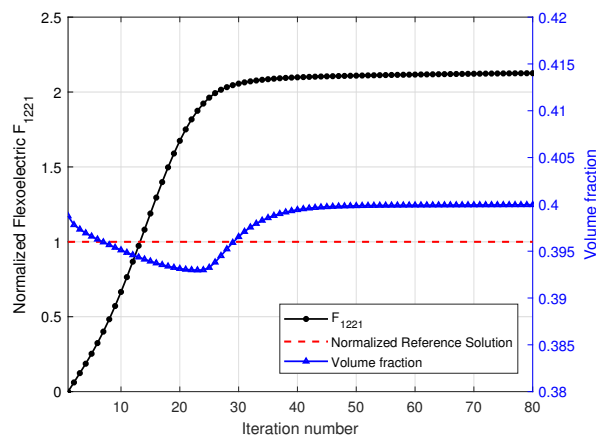

(c)

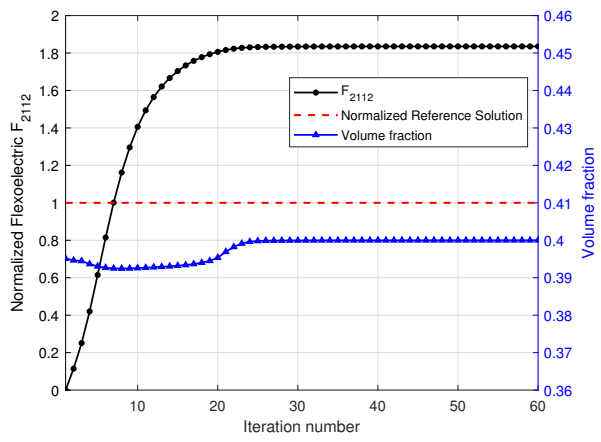

(b)

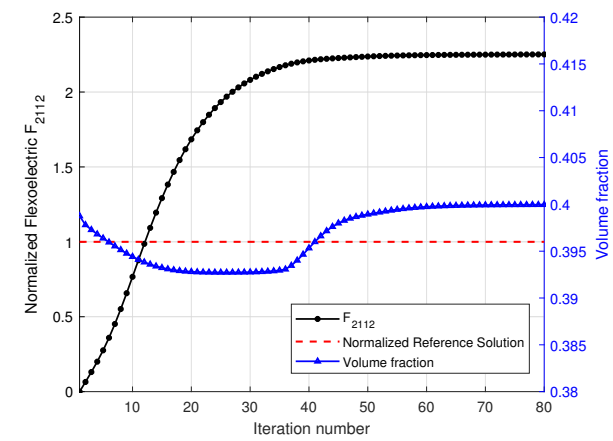

(d)

Figure 3: Topology optimization process with respect to normalized flexoelectric components and volume fractions for the PZT/PZT: (a) $\bar{F}_{1221}, 1 \times 1$ cells; (b) $\bar{F}_{2112}, 1 \times 1$ cells; (c) $\bar{F}_{1221}, 2 \times 2$ cells; (d) $\bar{F}_{2112}, 2 \times 2$ cells. 


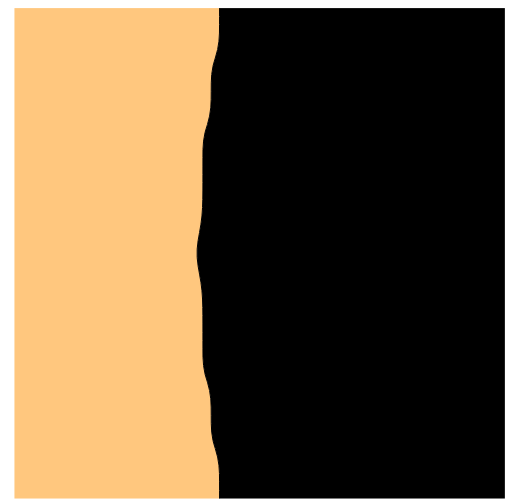

(a)

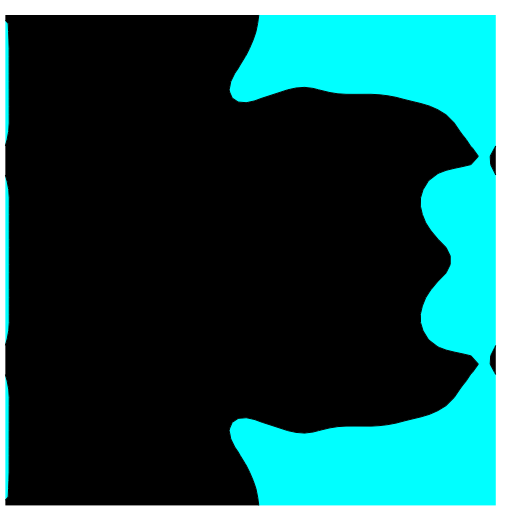

(c)

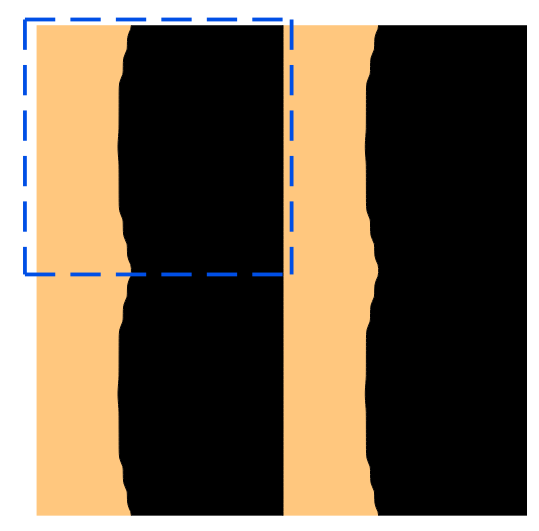

(b)

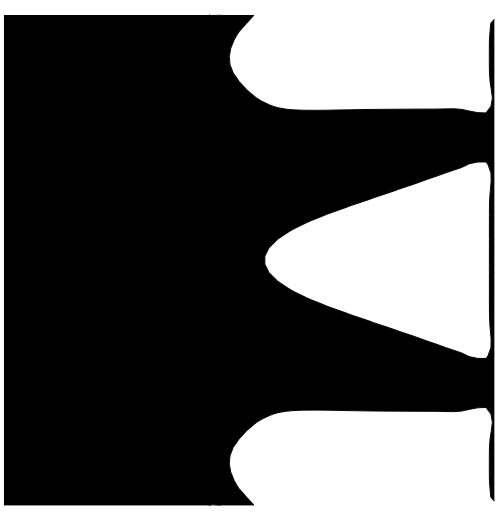

(d)

Figure 4: Optimal topology for $\bar{F}_{1221}$ : (a) PZT/PZT, $1 \times 1$ cells; (b)PZT/PZT, $2 \times 2$ cells; (c) PZT/polymer, $1 \times 1$ cells; (d) PZT/void, $1 \times 1$ cells. 


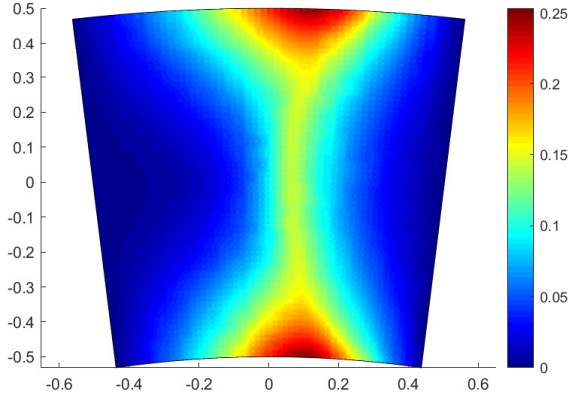

(a) $E_{2}(\mathbf{x})$

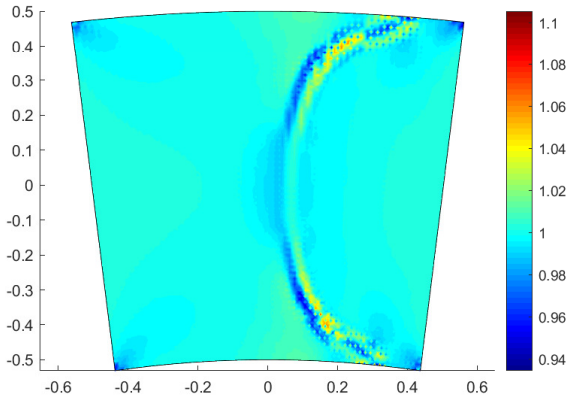

(b) $\nabla \varepsilon_{112}(\mathbf{x})$

Figure 5: Electric field ( $E_{2}$-component) and strain gradient $\left(\nabla \varepsilon_{112}\right.$-component within the PZT-PZToptimized microstructure corresponding to the optimized $\bar{F}_{2112}$ in Fig. 7 a).

the unit cell. We consider the optimized RVE of Fig $4 \mathrm{a}$ and use the same material parameters as in (58)- 61). Here again, the RVE is composed of $1 \times 1$ unit cell. The dimensions of the RVE are varied according to $\varepsilon=\ell / \ell_{0}$, where $\ell_{0}=1 \mathrm{~mm}$. We can see in Fig. 6 that the present model can capture the size effects of flexoelectric effective properties.

The electromechanical coefficients for the reference RVE with triangular inclusion are $\overline{\mathscr{K}}_{31}^{\text {ref }}=0.0929$ and $\overline{\mathscr{K}}_{32}^{\text {ref }}=0.0736$. For the optimized $\bar{F}_{1221}$, the coupling coefficients are found as $\overline{\mathscr{K}}_{31}=0.1141$ and $\overline{\mathscr{K}}_{32}=0.0903$, increasing respectively by $22.8 \%$ and $22.7 \%$. Similarly, for the optimized $\bar{F}_{2112}$, the coupling coefficients are found as $\overline{\mathscr{K}}_{31}=0.1183$ and $\overline{\mathscr{K}}_{32}=0.0937$, with each increasing by $27.3 \%$.

To gain more insight into the mechanisms driving the increase in the flexoelectric constants, we plot in Fig. 5 the local electric field component $E_{2}$ and local strain gradient component $\nabla \varepsilon_{112}$ within the optimized $F_{2112}$ unit cell shown in Fig. 7 (a) for a prescribed strain gradient $\bar{\nabla} \varepsilon_{112}=1 \mathrm{~m}^{-1}$. This is done as the value of the flexoelectric constant $F_{a j k l}$ depends on the polarization direction $a$, and the strain gradient $\nabla \bar{\varepsilon}_{j k l}$. We can observe that both the electric field and strain gradient are localized within the microstructure, roughly at the interface between the PZT matrix and PZT inclusion. Furthermore, the electric field is asymmetric with respect to the polarization direction, 


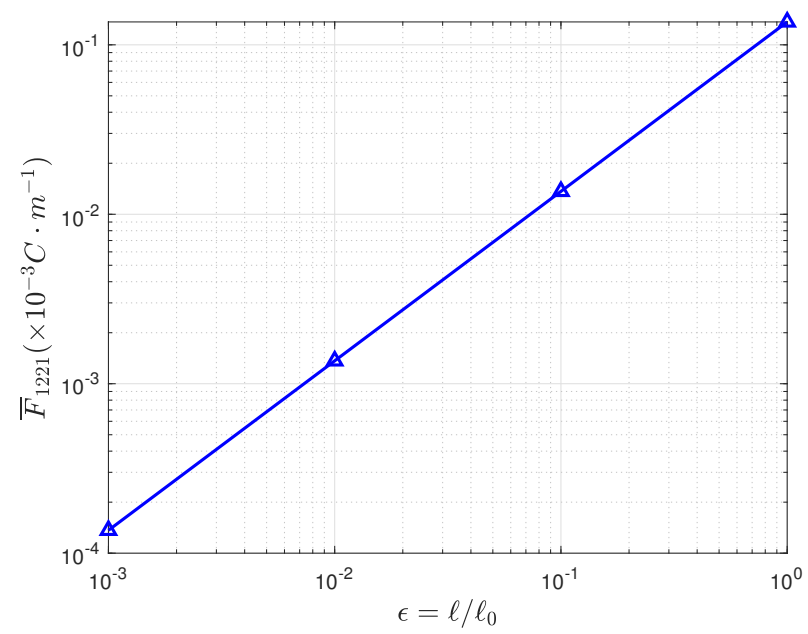

Figure 6: Size-dependent effective flexoelectric properties $\bar{F}_{1221}$ for the RVE with topology of Fig $4 \mathrm{a}$

which is required to obtain non-zero flexoelectric constants. While the strain gradient and electric field are both localized at the interface, the enhancements are not as large as for later examples using a hard/soft interface, and thus the enhancement in the optimized $\bar{F}_{2112}$, while excellent at $83.5 \%$, is smaller than the later optimized hard/soft structures.

To understand the enhancement in electromechanical coupling coefficient $\overline{\mathscr{K}}$, we examine the different terms contributing to it in Table 2. There, we find that for the PZT/PZT case, because the matrix and inclusion are comprised of the same material, the compliance $\overline{\mathbb{S}}$ and dielectric matrices $\overline{\mathbf{e}}$ have the same values. Therefore, the increase in electromechanical coupling $\overline{\mathscr{K}}$ for the hard/hard composite is entirely driven by the enhancement in effective RVE piezoelectric constants $\overline{\mathscr{D}}$.

Next, we investigate the influence of the volume fraction $f$ on the obtained geometries obtained by optimizing $\bar{F}_{1221}$ and $\bar{F}_{2112}$ in Figs. 8 and 9 , respectively. We first note that the volume fraction has a direct influence on the obtained geometry. When $f$ is around 0.5 , a simple layered structure is obtained. However, more asymmetric geometries with respect to the $y$-axis are induced for other volume fractions. In addition, the corresponding values of the optimized coefficients do not increase monotonically with 


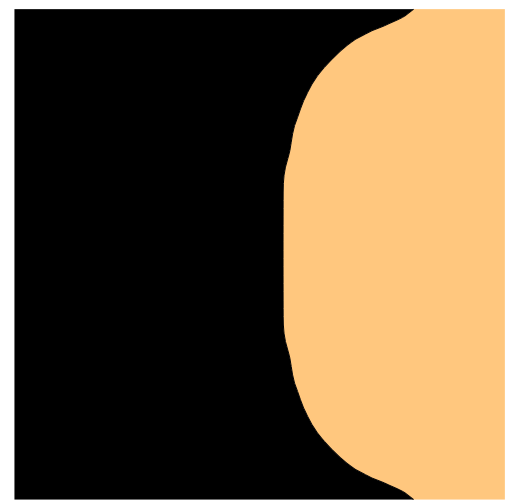

(a)

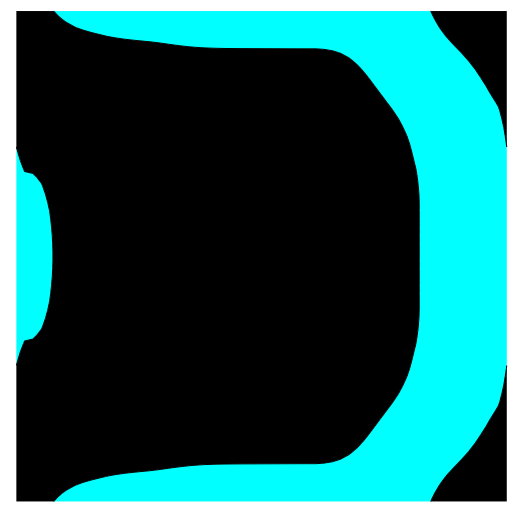

(c)

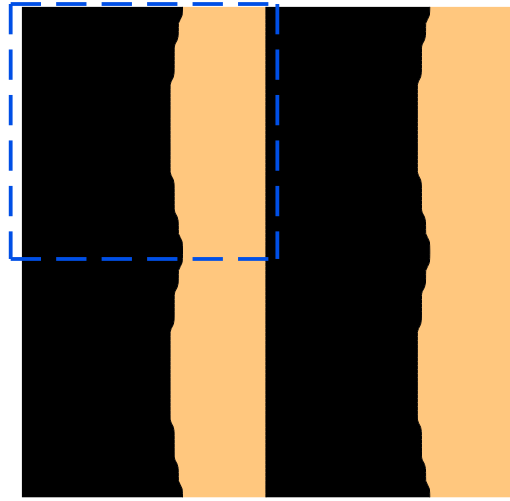

(b)

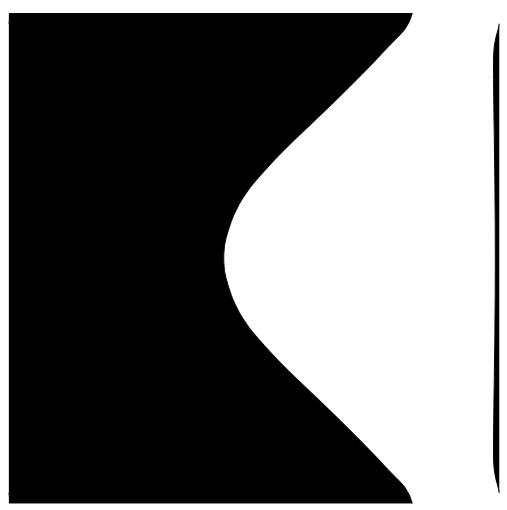

(d)

Figure 7: Optimal topology for $\bar{F}_{2112}$ : (a) PZT/PZT, $1 \times 1$ cells; (b)PZT/PZT, $2 \times 2$ cells; (c) PZT/polymer, $1 \times 1$ cells; (d) PZT/void, $1 \times 1$ cells. 
the volume fraction, but reach the largest value around $f=0.5$, leading to $\bar{F}_{1221}=1.43$ $\left(\times 10^{-4} \mathrm{C} \cdot \mathrm{m}^{-1}\right)$ and $\bar{F}_{2112}=2.767\left(\times 10^{-4} \mathrm{C} \cdot \mathrm{m}^{-1}\right)$. When the unit cell is homogeneous $(f=0$ and $f=1)$, the flexoelectric coefficients vanish.

It has been in shown in [66, 63] that in the present computational framework, the convergence of effective flexoelectric properties quickly converge with respect to the number of unit cells. Next, a $2 \times 2$ periodic repetition of unit cells is investigated to determine the influence of using more cells within the RVE for the calculations. Each unit cell is composed of $60 \times 60$ elements, thus $4 \times 60 \times 60$ elements are used in the $2 \times 2$ unit cells.

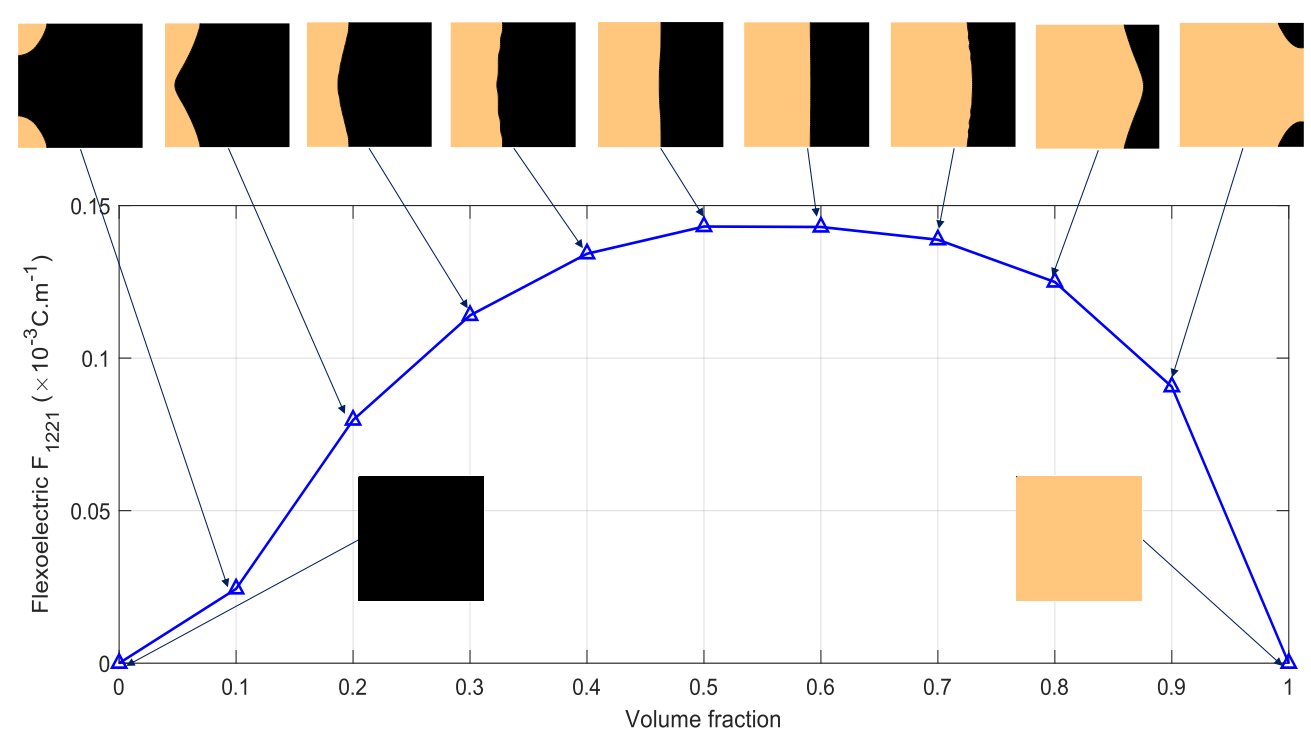

Figure 8: Optimal values of flexoelectric coefficient $\bar{F}_{1221}$ and corresponding topologies with respect to volume fraction of inclusion.

The optimized structures of the $2 \times 2$ periodic unit cell are obtained in Fig. $4 \mathrm{~b}$ and Fig. $7 \mathrm{~b}$ for $f=0.4$. In that case, the maximum values of the flexoelectric coefficients are $\bar{F}_{1221}=1.616\left(\times 10^{-4} \mathrm{C} \cdot \mathrm{m}^{-1}\right)$ and $\bar{F}_{2112}=3.298\left(\times 10^{-4} \mathrm{C} \cdot \mathrm{m}^{-1}\right)$. The coupling coefficients are obtained as $\overline{\mathscr{K}}_{31}=0.1449$ and $\overline{\mathscr{K}}_{32}=0.1147$ for optimized $\bar{F}_{1221}$, and $\overline{\mathscr{K}}_{31}=0.1169$ and $\overline{\mathscr{K}}_{32}=0.0925$ for optimized $\bar{F}_{2112}$. Then, a notable change is obtained as compared to the $1 \times 1$ unit cell. However, the obtained topologies are very 


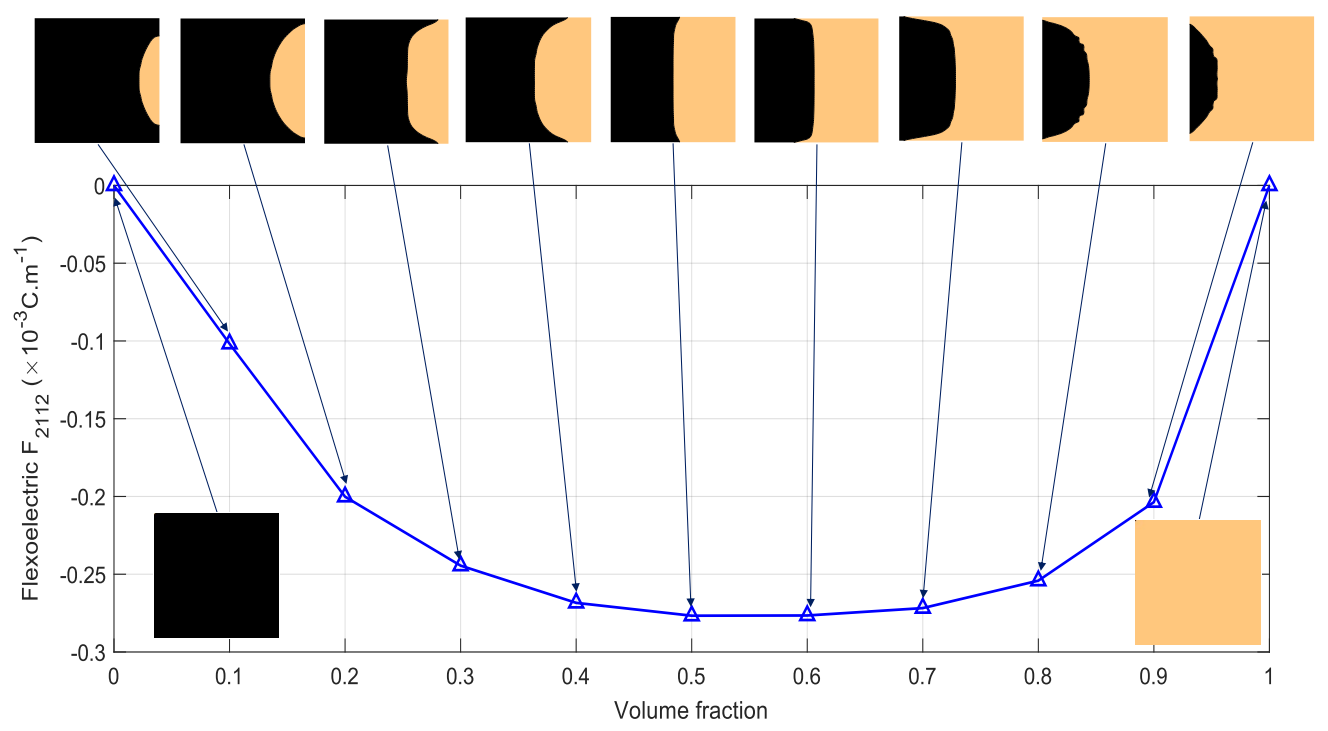

Figure 9: Optimal values of flexoelectric coefficient $\bar{F}_{2112}$ and corresponding topologies with respect to volume fraction of inclusion.

similar, which suggest that the topology optimization can be conducted on a single unit cell, while the effective properties can be estimated using more repeated unit cells. In our previous work [66] focusing on the homogenization of strain gradient elastic problems, we have shown that the present formulation leads to convergent properties with respect to the number of unit cells.

\subsection{Ceramic/doped piezoelectric polymer composite}

In our second example, we replace the misoriented and mechanically stiff PZT inclusion with a soft, dielectric, polymer inclusion (polyvinylidene fluoride, PVDF). The elastic, piezoelectric and dielectric properties for the polymer are given below. In comparison to the PZT properties in Eqs. [58)-(61), all of the polymer properties are 1-2 orders in magnitude lower than for PZT. Despite this, we shall demonstrate in this example that the potential of increased strain gradients that may be possible by using hard/soft composites can lead to effective flexoelectric constants and electromechanical coupling constants that can exceed those of the PZT/PZT composite in the previous 


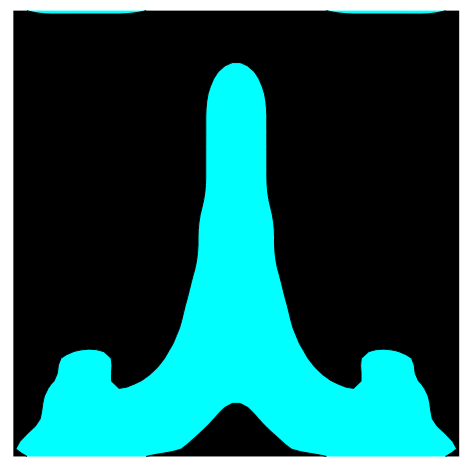

(a)

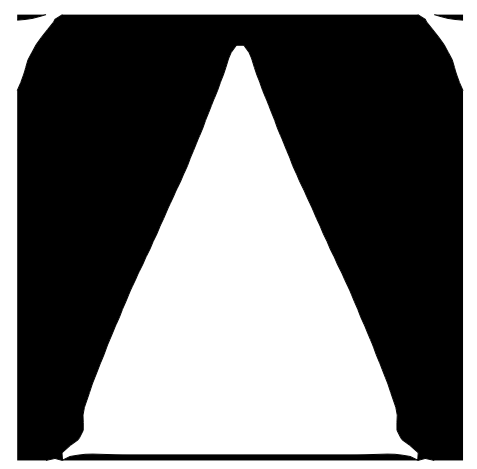

(b)

Figure 10: Optimal topology for $\bar{F}_{2221}$ : (a) PZT/polymer; (b) PZT/void.

example. The material parameters of matrix PZT are expressed in (58)-(60), while the material properties of PVDF are described in (62)-(64) [79].

$$
\begin{aligned}
& {\left[\mathbf{C}^{i}\right]=\left[\begin{array}{ccc}
6.066 & 3.911 & 0 \\
3.911 & 6.066 & 0 \\
0 & 0 & 1.078
\end{array}\right](G P a)} \\
& {\left[\boldsymbol{\alpha}^{i}\right]=\left[\begin{array}{cc}
0.025 & 0 \\
0 & 0.084
\end{array}\right]\left(C \cdot m^{-2}\right)} \\
& {\left[\mathscr{E}^{i}\right]=\left[\begin{array}{ccc}
0.1272 & 0.0873 & 0 \\
0 & 0 & 0
\end{array}\right]\left(n C \cdot m^{-1} \cdot V^{-1}\right)}
\end{aligned}
$$

We perform topology optimization of the PVDF inclusion with respect to the flexoelectric coefficients $\bar{F}_{1221}, \bar{F}_{2221}, \bar{F}_{1112}$ and $\bar{F}_{2112}$. To ensure that these results can be compared against the previous PZT/PZT results, we set the volume fraction of the PVDF inclusion to be $f=0.4$ for all cases. Similarly, the initial guess is set by $\rho_{e}=0.4, e=1,2, \ldots, N e=3600$. The periodic density conditions are considered here. The final optimal unit cells of the flexoelectric coefficients $\bar{F}_{1221}, \bar{F}_{2221}, \bar{F}_{1112}$ and $\bar{F}_{2112}$ are obtained in Figs. $4 \mathrm{c}, 10 \mathrm{a}, 11 \mathrm{a}$ and $7 \mathrm{c}$. In these figures, the cyan and black colors 


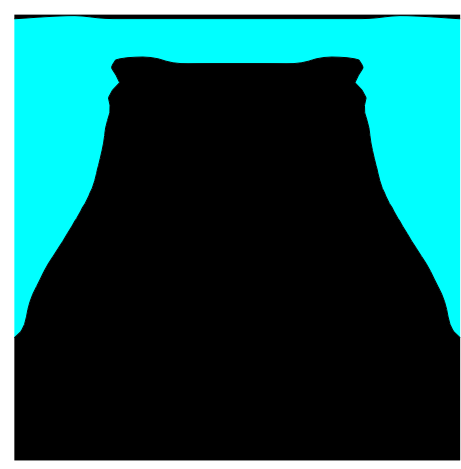

(a)

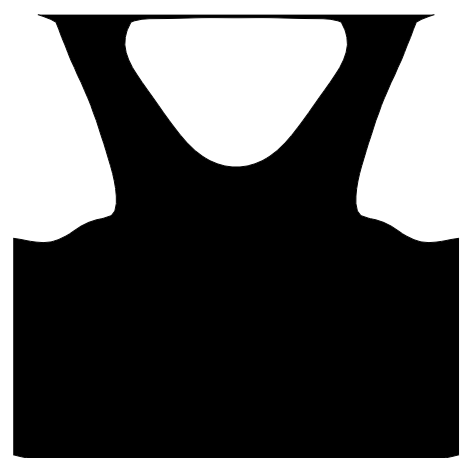

(b)

Figure 11: Optimal topology for $\bar{F}_{1112}$ : (a) PZT/polymer; (b) PZT/void

refer to the inclusion PVDF and matrix PZT, respectively. Iteration histories are shown in Fig. 12 The reference solutions calculated by a triangular PVDF inclusion as in Fig. 2] are shown in all cases.

We obtained four different optimized unit cells, and a significant improvement can be found compared to the reference triangular solutions. The optimal absolute values

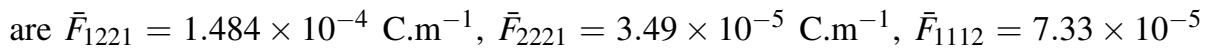
C.m ${ }^{-1}$ and $\bar{F}_{2112}=2.006 \times 10^{-4} \mathrm{C} . \mathrm{m}^{-1}$, which imply increases by $1462 \%, 113 \%$, $254 \%$ and $1431 \%$, respectively. Interestingly, despite being comprised of a polymer inclusion whose (elastic, piezoelectric, and dielectric) properties are all about two orders of magnitude smaller than the PZT matrix, the flexoelectric constants are quite similar to those obtained for the optimized PZT/PZT composites discussed previously, with significantly larger percentage enhancements.

To test the influence of the mesh, we compare the optimal topology configurations of PZT/PVDF composites with respect to $\bar{F}_{2221}$ using a regular mesh and an unstructured mesh. Both meshes contain 4-node elements and similar mesh densities. We can note from Fig. 13 that both topologies are almost identical, showing the meshindependence of the present formulation. The only slight differences come from the lack of periodicity in the unstructured mesh, leading to small perturbations near the boundaries. 


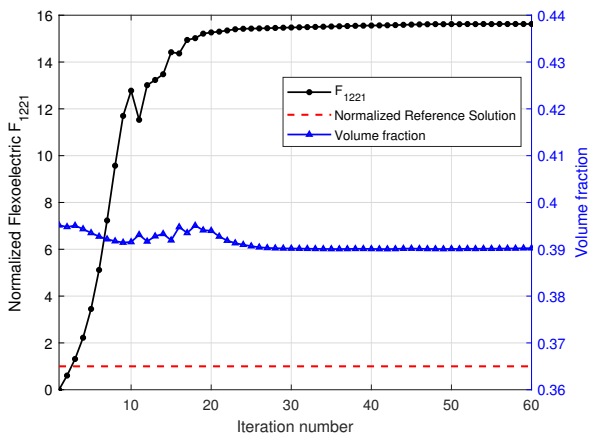

(a)

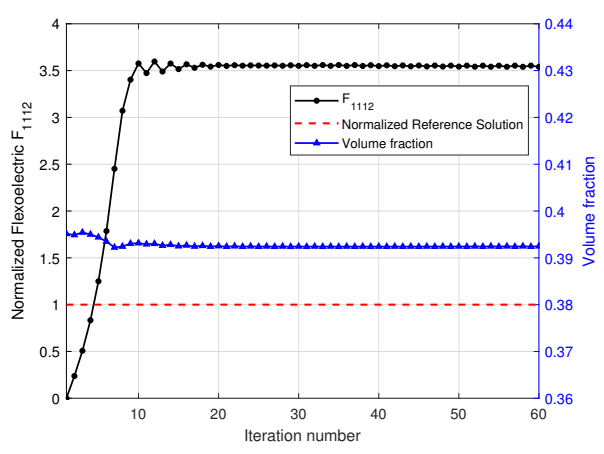

(c)

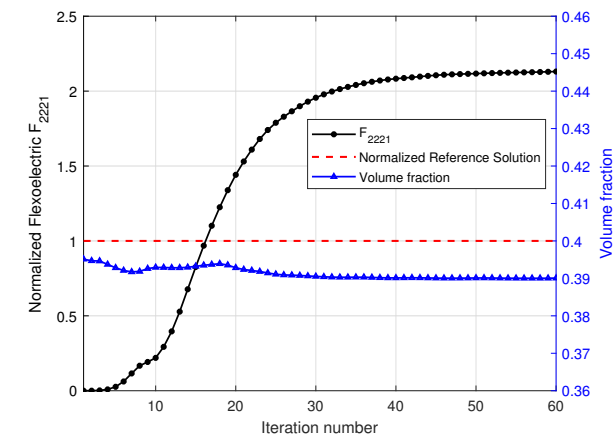

(b)

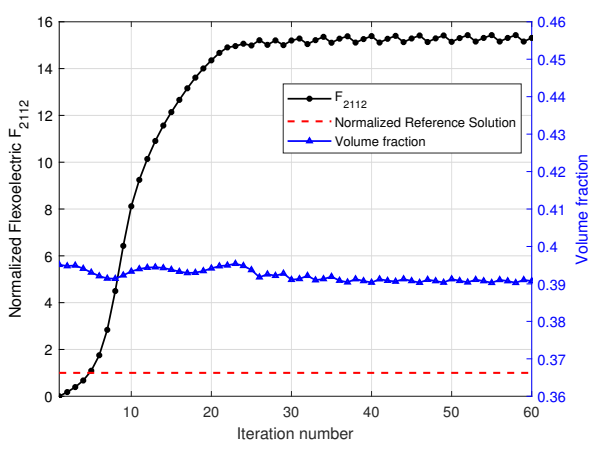

(d)

Figure 12: Topology optimization process with respect to normalized flexoelectric components and volume fractions for the PVDF/PZT: (a) $\bar{F}_{1221}$; (b) $\bar{F}_{2221}$; (c) $\bar{F}_{1112}$; (d) $\bar{F}_{2112}$. 
We then illustrate the flexoelectric effects on the optimized microstructures in Fig. 14. where the topology was optimized with respect to $\bar{F}_{2112}$ (geometry in Fig. 7 (c). We then apply an electric field along $y$ and allow the nodes along the boundary to move, except for one node that is constrained to avoid rigid-body motion. The deformations in the figure are exaggerated by a factor of 10 for ease of viewing, which demonstrates the bending deformation induced by the applied electric field.

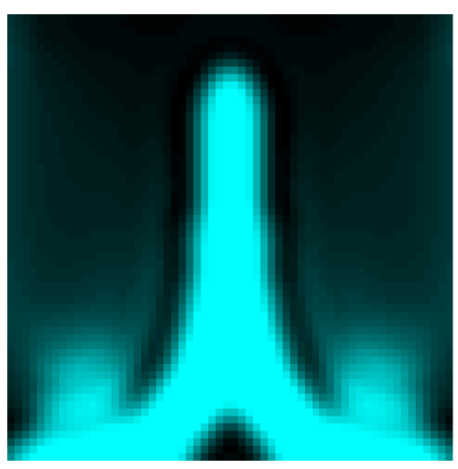

(a)

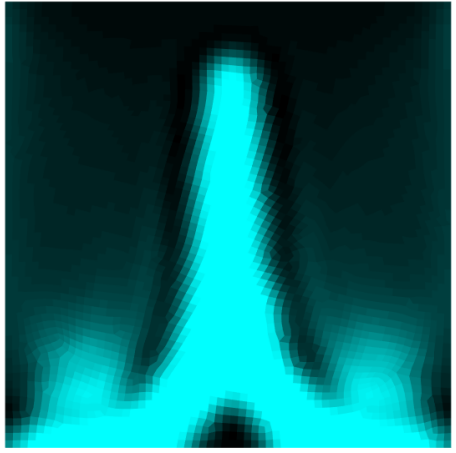

(b)

Figure 13: Optimal topology for $\bar{F}_{2221}$ on PZT/polymer: (a) with regular meshing; (b) with irregular meshing

The mechanisms for this effects can be seen in Fig. 15, where the electric field and strain gradient of the optimized unit cell for $\bar{F}_{2112}$ previously shown in Fig. 7 (c) are shown. In comparing the magnitudes of the electric field and strain gradient for the PZT/polymer RVE in Fig. 15 and the PZT/PZT RVE in Fig. 5, both the electric field and strain gradient for the hard/soft PZT/polymer case are 1-2 orders of magnitude larger than in the PZT/PZT case, which is reasonable given the curved hard/soft material boundary that exists within the RVE. Because the flexoelectric constants are dependent on the product of the electric field and strain gradient, this explains how the flexoelectric constants of the PZT/polymer case can rival and/or exceed those of the PZT/PZT case. as summarized in Table 1, despite being comprised of constituents with smaller physical properties.

The electromechanical coupling coefficients are also improved in the optimized designs. We obtain $\overline{\mathscr{K}}_{31}=0.409$ and $\overline{\mathscr{K}}_{32}=0.2694$ for optimized $\bar{F}_{1221}, \overline{\mathscr{K}}_{31}=0.1059$ 


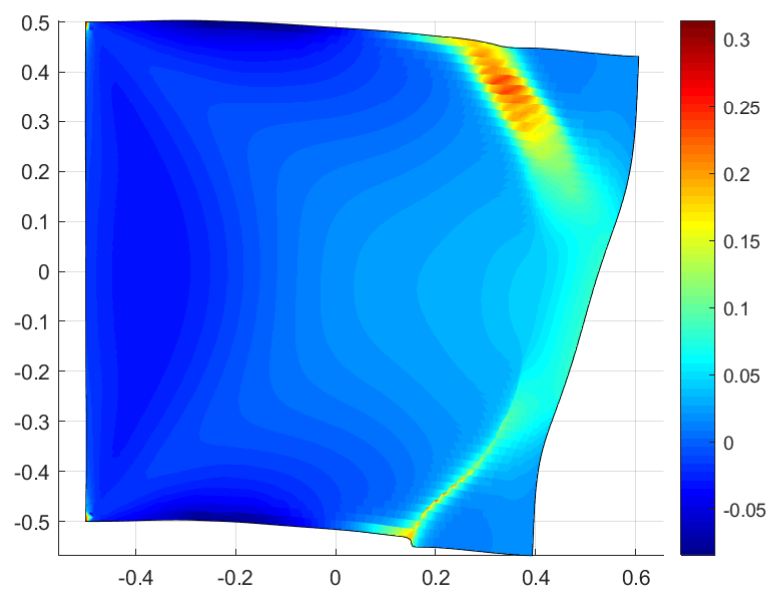

Figure 14: Deformation and strain $\varepsilon_{22}$ of optimized unit cell in Fig. 7c induced by electric filed $E_{2}$

$\overline{\mathscr{K}}_{32}=0.2444$ for optimized $\bar{F}_{2221}, \overline{\mathscr{K}}_{31}=0.4981$ and $\overline{\mathscr{K}}_{32}=0.3226$ for optimized $\bar{F}_{1112}$, while $\overline{\mathscr{K}}_{31}=0.0337$ and $\overline{\mathscr{K}}_{32}=0.3136$ for optimized $\bar{F}_{2112}$. In contrast with $\overline{\mathscr{K}}_{31}=0.3151$ and $\overline{\mathscr{K}}_{32}=0.067$ for triangular PVDF, the values of $\overline{\mathscr{K}}_{32}$ for the optimal unit cells are improved by $302.1 \%, 264.8 \%, 381.5 \%, 368.1 \%$, respectively, while $\overline{\mathscr{K}}_{31}$ for the optimal unit cells increases by $29.8 \%,-66.4 \%, 58.1 \%$ and $-89.3 \%$. We can note that the value of $\overline{\mathscr{K}}_{31}$ for optimized $\bar{F}_{2221}$ and $\bar{F}_{2112}$ decrease. However $\overline{\mathscr{K}}_{32}$ for all other optimized unit cells increase. For optimized $\bar{F}_{1221}$ and $\bar{F}_{1112}$, both electromechanical coefficients can be improved.

The mechanisms underlying the enhancement in electromechanical coupling coefficients corresponding to the PZT/polymer composites that maximize $\bar{F}_{2112}$ differ from those previously discussed for the PZT/PZT composites. In examining the contributions to the coupling coefficient $\overline{\mathscr{K}}$ in Table 2, we see that due to the multiple materials that comprise the RVE, all of the effective properties, i.e. compliance $\overline{\mathbb{S}}$, dielectric $\overline{\mathbf{e}}$ and piezoelectric $\overline{\mathscr{D}}$ change during the RVE optimization. For the $\overline{\mathscr{K}}_{31}$ constant, a significant decrease during optimization is found, which is driven by the significant decrease in the corresponding piezoelectric $\overline{\mathscr{D}}_{31}$ constant.

For the $\overline{\mathscr{K}}_{32}$ constant, a nearly five-fold increase is observed during optimization. Some of this is due to the doubling of the $\overline{\mathscr{D}}_{32}$ piezoelectric constant. However, the 


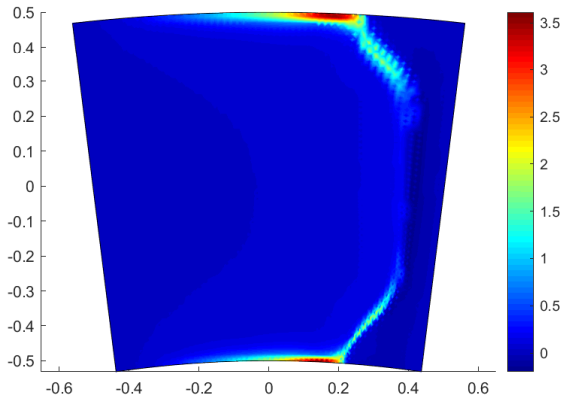

(a) $E_{2}(\mathbf{x})$

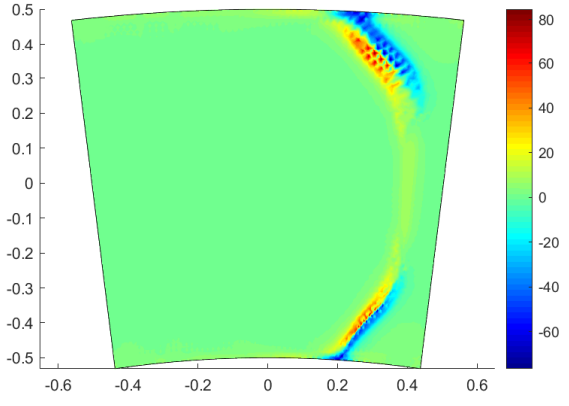

(b) $\nabla \varepsilon_{112}(\mathbf{x})$

Figure 15: Electric field ( $E_{2}$-component) and strain gradient ( $\nabla \varepsilon_{112}$-component) within the PZT-PVDFoptimized microstructure shown in Fig. 7 c).

optimization also leads to an increase in the dielectric properties $\overline{\mathbf{e}}$, and a decrease in the compliance $\overline{\mathbb{S}}$, as shown in Table 2. The increase in effective piezoelectric and dielectric properties are related to the enhanced localized electric field shown in Fig. 15. while the enhanced strain gradient shown in Fig. 15 is connected to the reduction in compliance. Thus, for the PZT/polymer RVE, it is this subtle interplay between the electrical, mechanical, and electromechanical properties that leads to the increase in electromechanical coupling.

\subsection{Heterogeneous porous microstructure}

In our final example, we consider a unit cell composed of a piezoelectric material with properties described by Eqs. (58)-(60), while the second phase is void. The flexoelectric coefficients $\bar{F}_{1221}, \bar{F}_{2221}, \bar{F}_{1112}$ and $\bar{F}_{2112}$ are considered. To model the void phase, soft properties are chosen for the void as $\left[C^{\text {void }}\right]=10^{-9} \times\left[C^{m}\right],\left[\mathscr{E}^{\text {void }}\right]=$ $10^{-9} \times\left[\mathscr{E}^{m}\right]$ and $\left[\alpha^{\text {void }}\right]=10^{-9} \times\left[\alpha^{m}\right]$.

The optimization is performed with respect to the different flexoelectric coefficients independently. In each case, the optimization process converges in roughly 80 iterations. Here, the volume fraction of the solid phase is constrained to $f=0.6$, such that the void (inclusion) volume fraction is 0.4 , the same as for the PZT/PZT and 


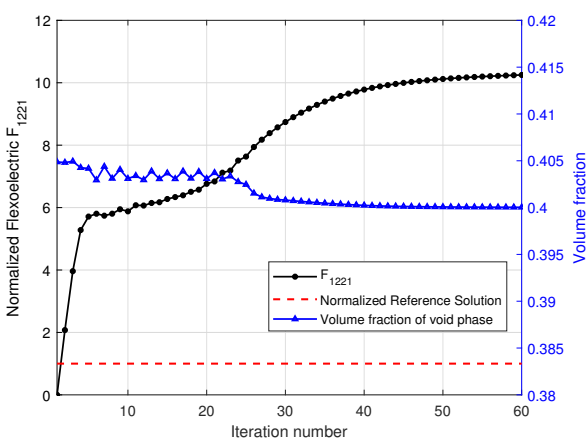

(a)

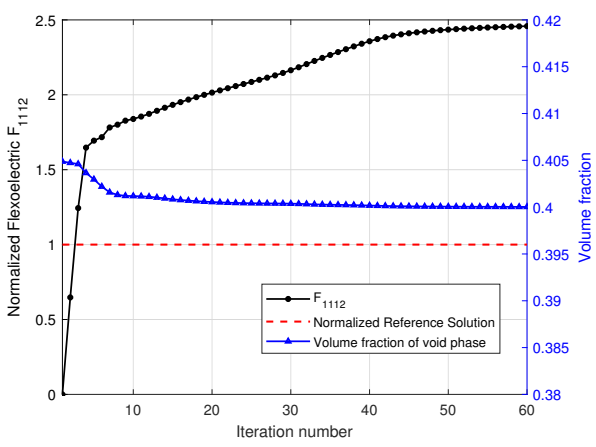

(c)

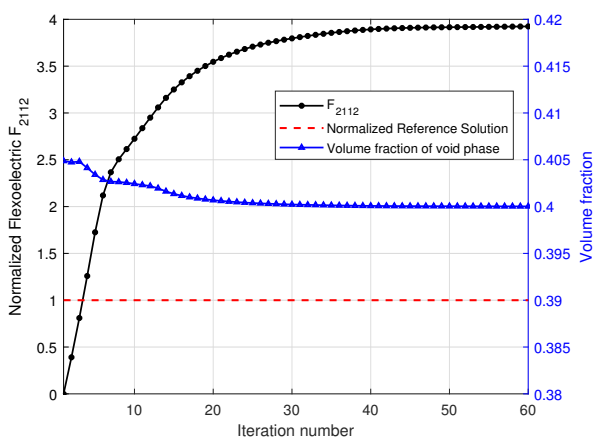

(b)

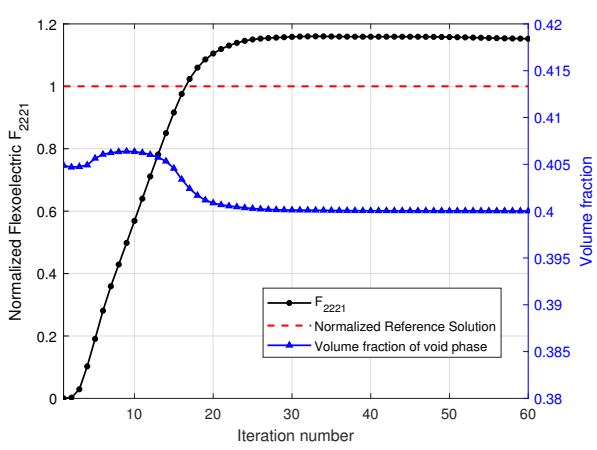

(d)

Figure 16: Topology optimization process with respect to normalized flexoelectric components and volume fractions for the PZT/void: (a) $\bar{F}_{1221}$; (b) $\bar{F}_{2112}$; (c) $\bar{F}_{1112}$; (d) $\bar{F}_{2221}$.

PZT/polymer composites. The initial design is a homogeneous unit cell with densities $\rho_{e}=0.6(e=1, \ldots, N e=3600)$. Periodic density conditions are used here. The final optimal design for the coefficients $\bar{F}_{1221}, \bar{F}_{2221}, \bar{F}_{1112}$ and $\bar{F}_{2112}$ are summarized in Figs. 4d, 7d, 11b and 10b, while their iteration histories are shown in Fig. 16 The reference solutions obtained by a triangular void as in Fig. 2 are reported in each case. We can see that four different optimized design are obtained for the different coefficients. It is interesting to note that the obtained geometries obtained by optimizing $\bar{F}_{1221}$ and $\bar{F}_{2112}$ have the same symmetry, as well as $\bar{F}_{2221}$ and $\bar{F}_{1112}$. In addition, we can note that even though the materials are different, the topologies obtained for the same optimized component can show significant similarities (see e.g. Figs. 10a and 10b 
The obtained absolute values are $\bar{F}_{1221}=7.99 \times 10^{-5}$ C.m ${ }^{-1}, \bar{F}_{2112}=3.85 \times 10^{-5}$ C.m ${ }^{-1}, \bar{F}_{1112}=2.36 \times 10^{-5}$ C.m ${ }^{-1}$ and $\bar{F}_{2221}=1.15 \times 10^{-5}$ C.m ${ }^{-1}$. In contrast with the flexoelectric properties of the unit cell with triangular void, we get a very large gain in the optimized structures for the components of $\bar{F}_{1221}, \bar{F}_{2112}$ and $\bar{F}_{1112}$, which are improved by $924 \%, 293 \%$ and $145 \%$, respectively. However, only an increase by $15 \%$ for $\bar{F}_{2221}$ is obtained, and it has the similar topology as the reference triangular unit cells. We obtain $\overline{\mathscr{K}}_{31}=0.4175$ and $\overline{\mathscr{K}}_{32}=0.2226$ for optimized $\bar{F}_{1221}, \overline{\mathscr{K}}_{31}=0.2086$ and $\overline{\mathscr{K}}_{32}=0.2064$ for optimized $\bar{F}_{2221}, \overline{\mathscr{K}}_{31}=0.3038$ and $\overline{\mathscr{K}}_{32}=0.1657$ for optimized $\bar{F}_{1112}$, while we have $\overline{\mathscr{K}}_{31}=0.2940$ and $\overline{\mathscr{K}}_{32}=0.2833$ for optimized $\bar{F}_{2112}$.

We show in Fig. 17 the electric field and strain gradient for corresponding to the optimized PZT/void microstructure in Fig. 7 that maximizes $\bar{F}_{2112}$. Similar to the PZT/polymer case in Fig. 15, the electric field and strain gradient are largest around the PZT/void interface, though the magnitude of each is smaller than in the PZT/polymer case. For that reason, the resulting flexoelectric constants for the PZT/void RVEs are smaller than the PZT/polymer and PZT/PZT RVEs, as summarized in Table 1 . The mechanism for the changes in electromechanical coupling are also similar to the PZT/polymer case. Specifically, localized electric field-driven increases along the PZT/void boundary lead to enhancements in the effective piezoelectric and dielectric properties, while the enhanced strain gradient is related to the reduction in compliance, with the interplay resulting in an increase in $\overline{\mathscr{K}}_{31}$ and an increase in $\overline{\mathscr{K}}_{32}$.

\subsection{Summary of results}

We summarize in Table 1 the optimal values for flexoelectric coefficients found in the different composites. For reference, the values are compared with the flexoelectric coefficient of $\mathrm{BaTiO}_{3}$ [80]. As can be seen, the RVE-based topology optimization approach leads to unit cells whose effective flexoelectric constants can exceed, by significant amounts, the flexoelectric properties of $\mathrm{BaTiO}_{3}$, as driven by the different electromechanical mechanisms discussed previously. 


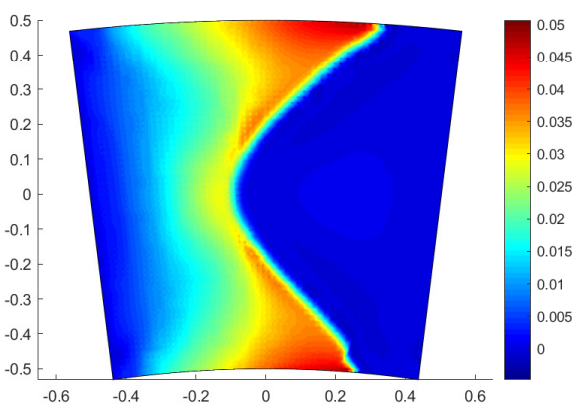

(a) $E_{2}(\mathbf{x})$

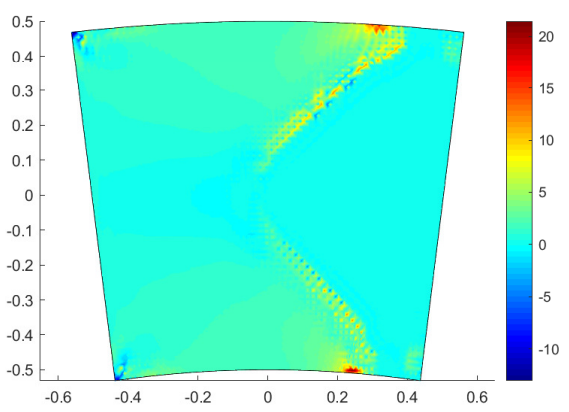

(b) $\nabla \varepsilon_{112}(\mathbf{x})$

Figure 17: Electric field ( $E_{2}$-component) and strain gradient $\left(\nabla \varepsilon_{112}\right)$-component within the PZT-voidoptimized microstructure in Fig. 7 (d).

Table 1: Optimized flexoelectric coefficients for PZT/PZT, PZT/PVDF and porous PZT composites.

\begin{tabular}{lllll}
\hline & $\bar{F}_{1221}$ & $\bar{F}_{2112}$ & $\bar{F}_{2221}$ & $\bar{F}_{1112}$ \\
\hline PZT/PZT & $136 \mu \mathrm{C} / \mathrm{m}$ & $268 \mu \mathrm{C} / \mathrm{m}$ & & \\
PZT/PVDF & $148 \mu \mathrm{C} / \mathrm{m}$ & $200 \mu \mathrm{C} / \mathrm{m}$ & $35 \mu \mathrm{C} / \mathrm{m}$ & $73 \mu \mathrm{C} / \mathrm{m}$ \\
Porous PZT & $80 \mu \mathrm{C} / \mathrm{m}$ & $38 \mu \mathrm{C} / \mathrm{m}$ & $11 \mu \mathrm{C} / \mathrm{m}$ & $23 \mu \mathrm{C} / \mathrm{m}$ \\
$\mathrm{BaTiO}_{3}$ [80] & $10-50 \mu \mathrm{C} / \mathrm{m}$ & & & \\
\hline
\end{tabular}




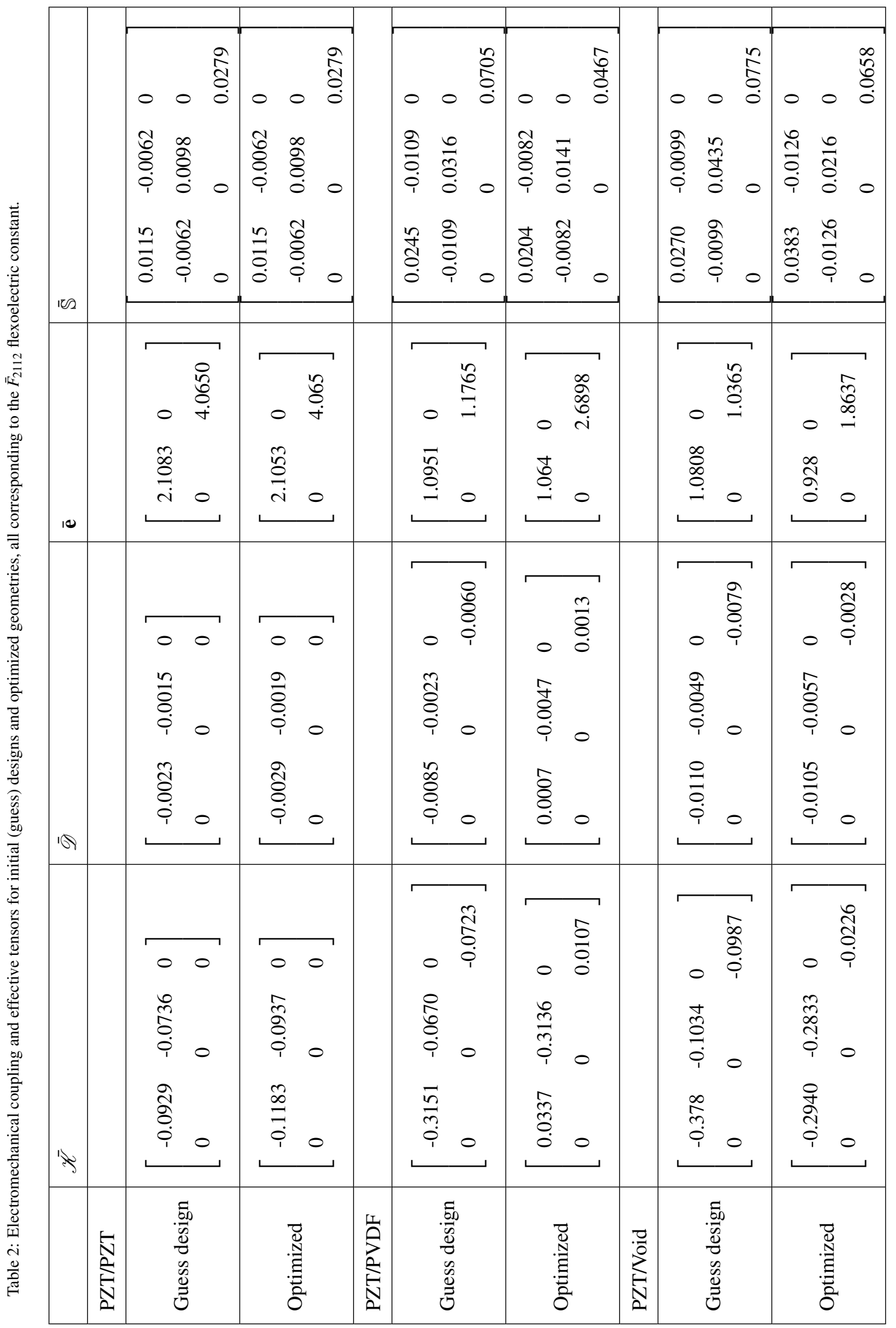




\section{Conclusion}

In this work, a topology optimization framework has been proposed to maximize the effective flexoelectric properties of composites made of piezoelectric phases. The originality of the present work is the use of a homogenization method to estimate the flexoelectric properties from the distribution of local phases in a Representative Volume Element (RVE), which precludes the necessity of optimizing the entire structure. A SIMP method was used to solve the topology optimization problem, where the absolute values of the flexoelectric tensor are maximized under the constraint of a constant volume fraction of inclusion. Results show that on several cases (piezo-piezo, piezopolymer and porous piezo-composites), the present scheme allows increasing the effective flexoelectric properties between 2 and 15 times as compared to a naive "guess" design.

We found different mechanisms to enhancing the flexoelectric properties, and the electromechanical coupling. Specifically, piezo-piezo (hard/hard) composites generated an enhanced electromechanical response through enhancement of their effective piezoelectric properties. In contrast, piezo-polymer (hard/soft) composites generated an enhanced electromechanical response through an interplay of enhanced electromechanical (piezoelectric) and electrical (dielectric) properties, and reduced mechanical compliance, which result from significantly enhanced local electric fields and strain gradients along the hard/soft interface. We believe that the present framework has the potential to design high-performance flexoelectric components for use e.g. in energy harvesting systems, sensors or actuators without the need for materials exhibiting intrinsically high flexoelectricity.

\section{Acknowledgement}

X.C gratefully acknowledges the funding from Project 31801-160170002, Graduate School, Central South University, China. 


\section{Appendix A. Numerical calculation of the effective tensors}

The $2 \mathrm{D}$ vector form associated with the components of strain gradient tensor $\nabla \varepsilon$ can be defined as:

$$
[\nabla \varepsilon]=\left[\begin{array}{c}
\nabla \varepsilon_{111} \\
\nabla \varepsilon_{221} \\
2 \nabla \varepsilon_{122} \\
\nabla \varepsilon_{222} \\
\nabla \varepsilon_{112} \\
2 \nabla \varepsilon_{121}
\end{array}\right]=\left[\begin{array}{c}
\frac{\partial^{2} u_{1}}{\partial x_{1}^{2}} \\
\frac{\partial^{2} u_{2}}{\partial x_{1} \partial x_{2}} \\
\frac{\partial^{2} u_{1}}{\partial x_{2}^{2}}+\frac{\partial^{2} u_{2}}{\partial x_{1} \partial x_{2}} \\
\frac{\partial^{2} u_{2}}{\partial x_{2}^{2}} \\
\frac{\partial^{2} u_{1}}{\partial x_{1} \partial x_{2}} \\
\frac{\partial^{2} u_{1}}{\partial x_{1} \partial x_{2}}+\frac{\partial^{2} u_{2}}{\partial x_{1}^{2}}
\end{array}\right]
$$

where the symmetries of effective tensors $[\overline{\boldsymbol{\alpha}}],[\overline{\mathscr{E}}],[\overline{\mathbb{C}}],[\overline{\mathbb{F}}],[\overline{\mathbb{M}}]$ and $[\overline{\mathbb{G}}]$ are taken into account from

$$
\begin{aligned}
& \overline{\mathscr{E}}_{i j k}=\overline{\mathscr{E}}_{i k j}, \overline{\mathbb{F}}_{i j k l}=\overline{\mathbb{F}}_{i k j l} \\
& \overline{\mathbb{M}}_{i j k l m}=\overline{\mathbb{M}}_{j i k l m}=\overline{\mathbb{M}}_{i j l k m} \\
& \overline{\mathbb{C}}_{i j k l}=\overline{\mathbb{C}}_{k l i j}=\overline{\mathbb{C}}_{j i k l}=\overline{\mathbb{C}}_{i j l k} \\
& \overline{\mathbb{G}}_{i j k l m p}=\overline{\mathbb{G}}_{l m p i j k}=\overline{\mathbb{G}}_{j i k l m p}=\overline{\mathbb{G}}_{i j k m l p}
\end{aligned}
$$

The flexoelectric tensor is written in matrix form as

$$
[\overline{\mathbb{F}}]=\left[\begin{array}{cccccc}
\bar{F}_{1111} & \bar{F}_{1221} & \bar{F}_{1122} & \bar{F}_{1222} & \bar{F}_{1112} & \bar{F}_{1121} \\
\bar{F}_{2111} & \bar{F}_{2221} & \bar{F}_{2122} & \bar{F}_{2222} & \bar{F}_{2112} & \bar{F}_{2121}
\end{array}\right]
$$

The matrix forms for the other effective tensors can be found in [63]. After discretization, the local strain and electric fields defined respectively in Eqs. (11) and (12) can expressed as:

$$
\begin{aligned}
& {[\varepsilon(\mathbf{x})]=\mathbf{A}^{0}(\mathbf{x}): \bar{\varepsilon}+\mathbf{B}^{0}(\mathbf{x}) \cdot \overline{\mathbf{E}}+\left\{\mathbf{A}^{1}(\mathbf{x})-\mathbf{A}^{0}(\mathbf{x}) \otimes \mathbf{x}\right\}: \overline{\nabla \varepsilon}} \\
& \mathbf{E}(\mathbf{x})=\mathbf{D}^{0}(\mathbf{x}): \overline{\boldsymbol{\varepsilon}}+\mathbf{h}^{0}(\mathbf{x}) \cdot \overline{\mathbf{E}}+\left\{\mathbf{D}^{1}(\mathbf{x})-\mathbf{D}^{0}(\mathbf{x})\right\} \vdots \overline{\nabla \varepsilon}
\end{aligned}
$$

We define the above displacement and electric fields matrices as:

$$
\begin{aligned}
& \mathbf{U}_{u}=\left[\mathbf{u}^{1}, \mathbf{u}^{2}, \mathbf{u}^{3}\right] ; \mathbf{V}_{u}=\left[\mathbf{u}^{4}, \mathbf{u}^{5}\right] ; \mathbf{W}_{u}=\left[\mathbf{u}^{6}, \mathbf{u}^{6}, \mathbf{u}^{7}, \mathbf{u}^{8}, \mathbf{u}^{10}, \mathbf{u}^{11}\right] \\
& \mathbf{U}_{\phi}=\left[\phi^{1}, \phi^{2}, \phi^{3}\right] ; \mathbf{V}_{\phi}=\left[\phi^{4}, \phi^{5}\right] ; \mathbf{W}_{\phi}=\left[\phi^{6}, \phi^{7}, \phi^{8}, \phi^{9}, \phi^{10}, \phi^{11}\right]
\end{aligned}
$$




\begin{tabular}{|c||c|c|c|}
\hline Field & $\left(\bar{\varepsilon}_{11}, \bar{\varepsilon}_{22}, \bar{\varepsilon}_{12}\right)$ & $\left(\bar{E}_{1}, \bar{E}_{2}\right)$ & $\left(\overline{\nabla \varepsilon}_{111}, \overline{\nabla \varepsilon}_{221}, \overline{\nabla \varepsilon}_{122}, \overline{\nabla \varepsilon}_{222}, \overline{\nabla \varepsilon}_{112}, \overline{\nabla \varepsilon}_{121}\right)$ \\
\hline $\mathbf{u}^{1}, \phi^{1}$ & $(1,0,0)$ & $(0,0)$ & $(0,0,0,0,0,0)$ \\
\hline $\mathbf{u}^{2}, \phi^{2}$ & $(0,1,0)$ & $(0,0)$ & $(0,0,0,0,0,0)$ \\
\hline $\mathbf{u}^{3}, \phi^{3}$ & $\left(0,0, \frac{1}{2}\right)$ & $(0,0)$ & $(0,0,0,0,0,0)$ \\
\hline $\mathbf{u}^{4}, \phi^{4}$ & $(0,0,0)$ & $(1,0)$ & $(0,0,0,0,0,0)$ \\
\hline $\mathbf{u}^{5}, \phi^{5}$ & $(0,0,0)$ & $(0,1)$ & $(0,0,0,0,0,0)$ \\
\hline $\mathbf{u}^{6}, \phi^{6}$ & $(0,0,0)$ & $(0,0)$ & $(1,0,0,0,0,0)$ \\
\hline $\mathbf{u}^{7}, \phi^{7}$ & $(0,0,0)$ & $(0,0)$ & $(0,1,0,0,0,0)$ \\
\hline $\mathbf{u}^{8}, \phi^{8}$ & $(0,0,0)$ & $(0,0)$ & $(0,0,1,0,0,0)$ \\
\hline $\mathbf{u}^{9}, \phi^{9}$ & $(0,0,0)$ & $(0,0)$ & $(0,0,0,1,0,0)$ \\
\hline $\mathbf{u}^{10}, \phi^{10}$ & $(0,0,0)$ & $(0,0)$ & $(0,0,0,0,1,0)$ \\
\hline $\mathbf{u}^{11}, \phi^{11}$ & $(0,0,0)$ & $(0,0)$ & $(0,0,0,0,0,1)$ \\
\hline
\end{tabular}

Table A.3: Elementary solution corresponding to the activated strain, electric potential and strain gradient components

and

$$
\begin{aligned}
\boldsymbol{W}_{u}^{x} & =\left[x \mathbf{u}^{1}, y \mathbf{u}^{1}, x \mathbf{u}^{2}, y \mathbf{u}^{2}, x \mathbf{u}^{3}, y \mathbf{u}^{3}\right] \\
\mathbf{W}_{\phi}^{x} & =\left[x \phi^{1}, y \phi^{1}, x \phi^{2}, y \phi^{2}, x \phi^{3}, y \phi^{3}\right]
\end{aligned}
$$

The displacement fields $\mathbf{u}^{i}$ and the electric fields $\phi^{i}$ are the vector columns containing respectively the nodal displacement and electric potentials solution of the localization problems Eq. (7)-(8)-(4)-(5) with the boundary conditions described in Table A.3

In terms of the above definition and finite element discretization, we obtain:

$$
\begin{array}{ll}
\mathbf{A}^{0}(\mathbf{x})=\mathbf{B}_{u}(\mathbf{x}) \mathbf{U}_{u} ; & \mathbf{B}^{0}(\mathbf{x})=\mathbf{B}_{u}(\mathbf{x}) \mathbf{V}_{u} \\
\mathbf{A}^{1}(\mathbf{x})=\mathbf{B}_{u}(\mathbf{x}) \mathbf{W}_{u} ; & \mathbf{A}_{x}^{0}(\mathbf{x})=\mathbf{B}_{u}(\mathbf{x}) \mathbf{W}_{u}^{x}
\end{array}
$$

and

$$
\begin{array}{ll}
\mathbf{D}^{0}(\mathbf{x})=-\mathbf{B}_{\phi}(\mathbf{x}) \mathbf{U}_{\phi} ; & \mathbf{h}^{0}(\mathbf{x})=-\mathbf{B}_{\phi}(\mathbf{x}) \mathbf{V}_{\phi} \\
\mathbf{D}^{0}(\mathbf{x})=-\mathbf{B}_{\phi}(\mathbf{x}) \mathbf{W}_{\phi} ; & \mathbf{D}_{x}^{0}(\mathbf{x})=-\mathbf{B}_{\phi}(\mathbf{x}) \mathbf{W}_{\phi}^{x}
\end{array}
$$


By introducing Eqs. A.7) and A.8 into Eqs. (33)- 36 , we can obtain the discretization forms of the six effective tensors. In the following, only the interesting fourth-order effective flexoelectric tensor is presented. The effective flexoelectric tensor is expressed as:

$$
\begin{aligned}
{[\overline{\mathbb{F}}]=} & \left\langle\left(\mathbf{B}^{0}(\mathbf{x})\right)^{T}: \mathbb{C}(\mathbf{x}): \tilde{\mathbf{A}}^{1}(\mathbf{x})-\left(\mathbf{h}^{0}(\mathbf{x})\right)^{T} \cdot \mathscr{E}(\mathbf{x}): \tilde{\mathbf{A}}^{1}(\mathbf{x})\right. \\
& \left.-\left(\mathbf{B}^{0}(\mathbf{x})\right)^{T}: \mathscr{E}^{T}(\mathbf{x}) \cdot \tilde{\mathbf{D}}^{1}(\mathbf{x})-\left(\mathbf{h}^{0}(\mathbf{x})\right)^{T} \cdot \boldsymbol{\alpha}(\mathbf{x}) \cdot \tilde{\mathbf{D}}^{1}(\mathbf{x})\right\rangle
\end{aligned}
$$

\section{Appendix B. Expressions of body forces in the localization problem}

One obvious issue with condition (5) arises when considering a homogeneous RVE

characterized by elastic and piezoelectric tensors $\mathbb{C}^{1}$ and $\mathscr{E} 1$. In that case, and for $\bar{\varepsilon}=0$ and $\overline{\mathbf{E}}=0$, it is expected that the local strain solutions within the RVE should be equal to:

$$
\varepsilon(\mathbf{x})=\overline{\nabla \varepsilon} \cdot \mathbf{x} \forall \mathbf{x} \in \Omega
$$

However generally (B.1) is not a statically admissible solution for boundary conditions (5) since:

$$
\nabla \cdot\left(\mathbb{C}^{1}:[\overline{\nabla \varepsilon} \mathbf{x}]\right) \neq 0
$$

and

$$
\nabla \cdot\left(\mathscr{E}^{1}:[\overline{\nabla \varepsilon} \mathbf{x}]\right) \neq 0
$$

The inequalities B.2 $-\bar{B}$ B.3 hold because in the present work $\overline{\nabla \varepsilon}$ can be chosen arbitrarily. Therefore, as observed in [81, 82], fluctuations remain even when the local continuum is homogeneous, leading to persistent non-physical gradient effects. Indeed, when the local medium is Cauchy homogeneous, there is no dependence on an internal length and the overall medium cannot be of generalized type. To cure this problem, and following the analysis conducted in [83, 84], we propose to prescribe body forces in 
addition to QBC (5) to enforce a constant strain-gradient within the RVE when the material is homogeneous. The new localization problem involves solving the equilibrium equation:

$$
\nabla \cdot \sigma(\mathbf{x})=\mathbf{f}(\overline{\nabla \varepsilon}) \forall \mathbf{x} \in \Omega
$$

and

$$
\nabla \cdot \mathbf{d}(\mathbf{x})=\mathbf{r}(\overline{\nabla \varepsilon}) \forall \mathbf{x} \in \Omega
$$

where

$$
\mathbf{f}(\overline{\nabla \varepsilon})=\nabla \cdot\left(\mathbb{C}^{0}(\mathbf{x}):(\overline{\nabla \varepsilon} \cdot \mathbf{x})\right)
$$

and

$$
r(\overline{\nabla \varepsilon})=\nabla \cdot\left(\mathscr{E} C^{0}(\mathbf{x}):(\overline{\nabla \varepsilon} \cdot \mathbf{x})\right)
$$

In the definition of $\mathbf{f}$ and $r, \mathbb{C}^{0}(\mathbf{x})$ and $\mathscr{E}^{0}(\mathbf{x})$ are arbitrary elastic and piezoelectric tensor fields which have to be specified. At this point, and without loss of generality, we assume a two-phase composite whose elastic properties are described by $\mathbb{C}^{1}$ and $\mathbb{C}^{2}$, and where piezoelectric properties are defined by $\mathscr{E}^{1}$ and $\mathscr{E}^{2}$; in which the phase 1 has the highest volume fraction. The RVE is piezoelectric- homogeneous if either (a) the volume fraction of phase 2 goes to zero, i.e. $f^{1} \rightarrow 1$, or (b) if the contrast between phase properties goes to one, i.e. $\left\|\mathbb{C}^{2}\right\| \rightarrow\left\|\mathbb{C}^{1}\right\|$ and $\left\|\mathscr{E}^{2}\right\| \rightarrow\left\|\mathscr{E}^{1}\right\|$. For each of these two conditions, the tensors $\mathbb{C}^{0}(\mathbf{x})$ and $\mathscr{E}^{0}(\mathbf{x})$ should satisfy:

$$
\mathbb{C}^{0}(\mathbf{x}) \rightarrow \mathbb{C}^{1} \text { if }\left\{\begin{array}{l}
f^{1} \rightarrow 1, \\
\text { or }\left\|\mathbb{C}^{2}\right\| \rightarrow\left\|\mathbb{C}^{1}\right\|
\end{array}\right.
$$

and

$$
\mathscr{E}^{0}(\mathbf{x}) \rightarrow \mathscr{E}^{1} \quad \text { if }\left\{\begin{array}{l}
f^{1} \rightarrow 1, \\
\text { or }\left\|\mathscr{E}^{2}\right\| \rightarrow\left\|\mathscr{E}^{1}\right\| .
\end{array}\right.
$$

Several choices are possible to respect conditions $(B .8)-(B .9)$, such as (among others): pointwise body force correction, $\mathbb{C}^{0}(\mathbf{x})=\mathbb{C}(\mathbf{x})$ and $\mathscr{E}^{0}(\mathbf{x})=\mathscr{E}(\mathbf{x})$, effective body force correction, $\mathbb{C}^{0}(\mathbf{x})=\overline{\mathbb{C}}$ and $\mathscr{E}^{0}(\mathbf{x})=\overline{\mathscr{E}}$, or null body force (standard QBC) 
$\mathbb{C}^{0}(\mathbf{x})=\mathbb{O}$ and $\mathscr{E}^{0}(\mathbf{x})=\mathbb{O}$. These different choices have been compared in the elastic case in [84], and there is still no definitive answer to the best choice. The standard solution is simple but induces the mentioned spurious strain gradient effects in the case of homogeneous domains, as discussed in [84]. The effective body forces solution is consistent with asymptotic analysis [83] and removes these spurious effects but induces divergence of effective properties in case of infinite contrasts of properties between phases. A more detailed discussion can be found in [84]. In spite of these remaining issues, we adopt the effective body forces solution in the present work. Then, defining $\mathbb{C}^{0}=\overline{\mathbb{C}}$ and $\mathscr{E}^{0}=\overline{\mathscr{E}}$ (defined respectively by Eqs. (33) and 35) and introducing them in (B.6) and (B.7), we obtain Eqs. (9) and (10).

\section{References}

[1] L. E. Cross, Flexoelectric effects: Charge separation in insulating solids subjected to elastic strain gradients, Journal of Materials Science 41 (1) (2006) 53-63. doi : $10.1007 / \mathrm{s} 10853-005-5916-6$.

[2] J. Y. Fu, W. Zhu, N. Li, L. E. Cross, Experimental studies of the converse flexoelectric effect induced by inhomogeneous electric field in a barium strontium titanate composition, Journal of Applied Physics 100 (2) (2006) 024112. doi: $10.1063 / 1.2219990$.

[3] W. Ma, L. Cross, Flexoelectric polarization of barium strontium titanate in the paraelectric state, Appl. Phys. Lett. 81(18) (2002) 3440-3442.

[4] P. Zubko, G. Catalan, A. Buckley, P. R. L. Welche, J. F. Scott, Strain-gradientinduced polarization in srtio 3 single crystals, Phys. Rev. Lett. 99 (2007) 167601. doi:10.1103/PhysRevLett.99.167601.

[5] W. Ma, L. Cross, Strain-gradient induced electric polarization in lead zirconate titanate ceramics, Appl. Phys. Lett. 82(19) (2003) 3923-3925.

[6] B. Chu, D. R. Salem, Flexoelectricity in several thermoplastic and thermosetting polymers, Applied Physics Letters 101 (10) (2012) 103905. doi:10.1063/1. 4750064 
[7] P. Zubko, G. Catalan, A. K. Tagantsev, Flexoelectric effect in solids, Annual Review of Materials Research 43 (1) (2013) 387-421. doi:10.1146/ annurev-matsci-071312-121634.

[8] Q. Deng, L. Liu, P. Sharma, Flexoelectricity in soft materials and biological membranes, Journal of the Mechanics and Physics of Solids 62 (2014) 209 - 227, sixtieth anniversary issue in honor of Professor Rodney Hill. doi: $10.1016 / j \cdot j m p s .2013 .09 .021$

[9] S. Kogan, Piezoelectric effect during inhomogeneous deformation and acoustic scattering of carriers in crystals, Soviet Physics-Solid State 5 (1964) 197 - 224.

[10] A. K. Tagantsev, Theory of flexoelectric effect in crystals, Soviet Physics JETP 61 (6) (1985) 1246.

[11] A. K. Tagantsev, Piezoelectricity and flexoelectricity in crystalline dielectrics, Physical Review B 34 (8) (1986) 5883-5889. doi:10.1103/PhysRevB.34. 5883.

[12] R. Maranganti, N. Sharma, P. Sharma, Electromechanical coupling in nonpiezoelectric materials due to nanoscale nonlocal size effects: Green's function solutions and embedded inclusions, Physical Review B 74 (1) (2006) 014110.

[13] N. D. Sharma, R. Maranganti, P. Sharma, On the possibility of piezoelectric nanocomposites without using piezoelectric materials, Journal of the Mechanics and Physics of Solids 55 (11) (2007) 2328-2350. doi:10.1016/j.jmps. 2007.03 .016 .

[14] W. Zhu, J. Y. Fu, N. Li, L. Cross, Piezoelectric composite based on the enhanced flexoelectric effects, Applied Physics Letters 89 (19) (2006) 192904. doi:10. $1063 / 1.2382740$

[15] M. S. Majdoub, P. Sharma, T. Cagin, Enhanced size-dependent piezoelectricity and elasticity in nanostructures due to the flexoelectric effect, Phys. Rev. B 77 (2008) 125424. doi:10.1103/PhysRevB.77.125424. 
[16] S. Shen, S. Hu, A theory of flexoelectricity with surface effect for elastic dielectrics, Journal of the Mechanics and Physics of Solids 58 (5) (2010) 665-677.

[17] S. Mao, P. K. Purohit, Insights into flexoelectric solids from strain-gradient elasticity, Journal of Applied Mechanics 81 (8) (2014).

[18] A. Abdollahi, C. Peco, D. Millán, M. Arroyo, I. Arias, Computational evaluation of the flexoelectric effect in dielectric solids, Journal of Applied Physics 116 (9) (2014) 093502. doi:10.1063/1.4893974

[19] A. Abdollahi, D. Millán, C. Peco, M. Arroyo, I. Arias, Revisiting pyramid compression to quantify flexoelectricity: A three-dimensional simulation study, Phys. Rev. B 91 (2015) 104103. doi:10.1103/PhysRevB.91.104103.

[20] Z. Zhang, D. Geng, X. Wang, Calculation of the piezoelectric and flexoelectric effect in nanowires using a decoupled finite element analysis method, Journal of Applied Physics 119 (2016) 154104.

[21] D. Codony, O. Marco, S. Fernández-Méndez, I. Arias, An immersed boundary hierarchical b-spline method for flexoelectricity, Computer Methods in Applied Mechanics and Engineering 354 (2019) 750-782.

[22] P. Yudin, A. Tagantsev, Fundamentals of flexoelextricity in solids, Nanotechnology 24 (2013) 432001.

[23] A. Tagantsev, Pyroelectric, piezoelectric, flexoelectric, and thermal polarization effects in ionic crystals, Soviet Physics Uspekhi 30 (7) (1987) 588.

[24] W. Ma, Flexoelectric charge separation and size dependent piezoelectricity in dielectric solids, Physica Status Solidi (b) 247 (1) (2010) 213-218.

[25] D. Lee, T. Noh, Giant flexoelectric effect through interfacial strain relaxation, Philosophical Transactions of the Royal Society A: Mathematical, Physical and Engineering Sciences 370 (1977) (2012) 4944-4957.

[26] T. Nguyen, S. Mao, Y.-W. Yeh, P. Purohit, M. McAlpine, Nanoscale flexoelectricity, Advanced Materials 25 (7) (2013) 946-974. 
[27] S. Krichen, P. Sharma, Flexoelectricity: A perspective on an unusual electromechanical coupling, Journal of Applied Mechanics 83 (3), 030801 (01 2016). doi:10.1115/1.4032378

[28] A. Rahmati, S. Bauer, P. Sharma, Nonlinear bending deformation of soft electrets and prospects for engineering flexoelectricity and transverse (d 31) piezoelectricity, Soft matter 15 (1) (2019) 127-148.

[29] E. Cholleti, A review on 3D printing of piezoelectric materials, in: IOP Conference Series: Materials Science and Engineering, Vol. 455, IOP Publishing, 2018, p. 012046.

[30] D. Guinovart-Sanjuán, J. Merodio, J. López-Realpozo, K. Vajravelu, R. Rodríguez-Ramos, R. Guinovart-Díaz, J. Bravo-Castillero, F. Sabina, Asymptotic homogenization applied to flexoelectric rods, Materials 12 (2) (2019) 232.

[31] S. Sidhardh, M. Ray, Effective properties of flexoelectric fiber-reinforced nanocomposite, Materials Today Communications 17 (2018) 114-123.

[32] V. Eremeyev, J.-F. Ganghoffer, V. Konopińska-Zmysłowska, N. Uglov, Flexoelectricity and apparent piezoelectricity of a pantographic micro-bar, International Journal of Engineering Science 149 (2020) 103213.

[33] P. Mohammadi, L. Liu, P. Sharma, A theory of flexoelectric membranes and effective properties of heterogeneous membranes, Journal of Applied Mechanics 81 (1) (2014).

[34] H. Chen, S. Zhang, A. Soh, W. Yin, Phase field modeling of flexoelectricity in solid dielectrics, Journal of Applied Physics 118 (3) (2015) 034106.

[35] M. P. Bendsøe, N. Kikuchi, Generating optimal topologies in structural design using a homogenization method, Computer Methods in Applied Mechanics and Engineering 71 (2) (1988) 197 - 224. doi:10.1016/0045-7825 (88) 90086-2.

[36] M. P. Bendsøe, Optimal shape design as a material distribution problem, Structural Optimization 1 (4) (1989) 193-202. doi:10.1007/BF01650949. 
[37] M. Zhou, G. Rozvany, The coc algorithm, part ii: Topological, geometrical and generalized shape optimization, Computer Methods in Applied Mechanics and Engineering 89 (1) (1991) 309 - 336, second World Congress on Computational Mechanics. doi:10.1016/0045-7825(91)90046-9.

[38] G. Allaire, F. Jouve, A.-M. Toader, A level-set method for shape optimization, Comptes Rendus Mathematique 334 (12) (2002) 1125 - 1130. doi:10.1016/ S1631-073X (02) 02412-3.

[39] M. Y. Wang, X. Wang, D. Guo, A level set method for structural topology optimization, Computer Methods in Applied Mechanics and Engineering 192 (1) (2003) 227 - 246. doi:/10.1016/S0045-7825(02)00559-5

[40] O. Sigmund, K. Maute, Topology optimization approaches, Structural and Multidisciplinary Optimization 48 (6) (2013) 1031-1055. doi:10.1007/ s00158-013-0978-6.

[41] Y. Xie, G. Steven, A simple evolutionary procedure for structural optimization, Computers and Structures 49 (5) (1993) 885 - 896. doi:10.1016/ 0045-7949(93)90035-C.

[42] X. Huang, Y. M. Xie, Bi-directional evolutionary topology optimization of continuum structures with one or multiple materials, Computational Mechanics 43 (3) (2009) 393-401. doi:10.1007/s00466-008-0312-0.

[43] O. Sigmund, Materials with prescribed constitutive parameters: an inverse homogenization problem, International Journal of Solids and Structures 31 (17) (1994) 2313-2329.

[44] O. Sigmund, S. Torquato, Design of materials with extreme thermal expansion using a three-phase topology optimization method, Journal of the Mechanics and Physics of Solids (1997). doi:10.1016/S0022-5096(96)00114-7.

[45] E. C. N. Silva, J. S. O. Fonseca, N. Kikuchi, Optimal design of piezoelectric microstructures, Computational Mechanics 19 (5) (1997) 397-410. doi: $10.1007 / \mathrm{s} 004660050188$ 
[46] F. Wang, O. Sigmund, J. Jensen, Design of materials with prescribed nonlinear properties, Journal of the Mechanics and Physics of Solids 69 (2014) 156 - 174. doi:10.1016/j.jmps.2014.05.003

[47] S. Nanthakumar, X. Zhuang, H. S. Park, T. Rabczuk, Topology optimization of flexoelectric structures, Journal of the Mechanics and Physics of Solids 105 (2017) 217 - 234. doi:10.1016/j.jmps.2017.05.010

[48] H. Ghasemi, H. S. Park, T. Rabczuk, A multi-material level set-based topology optimization of flexoelectric composites, Computer Methods in Applied Mechanics and Engineering 332 (2018) 47 - 62. doi:10.1016/j .cma.2017.12.005

[49] H. Ghasemi, H. S. Park, T. Rabczuk, A level-set based iga formulation for topology optimization of flexoelectric materials, Computer Methods in Applied Mechanics and Engineering 313 (2017) 239-258.

[50] H. Ghasemi, H. S. Park, N. Alajlan, T. Rabczuk, A computational framework for design and optimization of flexoelectric materials, International Journal of Computational Methods 17 (01) (2020) 1850097.

[51] M. P. Bendsoe, N. Kikuchi, Generating optimal topologies in structural design using a homogenization method (1988).

[52] M. P. Bendsøe, Optimal shape design as a material distribution problem, Structural optimization 1 (4) (1989) 193-202.

[53] G. I. Rozvany, M. Zhou, T. Birker, Generalized shape optimization without homogenization, Structural optimization 4 (3-4) (1992) 250-252.

[54] M. P. Bendsøe, O. Sigmund, Material interpolation schemes in topology optimization, Archive of applied mechanics 69 (9-10) (1999) 635-654.

[55] M. Y. Wang, X. Wang, D. Guo, A level set method for structural topology optimization, Computer methods in applied mechanics and engineering 192 (1-2) (2003) 227-246. 
[56] G. Allaire, F. Jouve, A.-M. Toader, Structural optimization using sensitivity analysis and a level-set method, Journal of computational physics 194 (1) (2004) 363393.

[57] Y. M. Xie, G. P. Steven, A simple evolutionary procedure for structural optimization, Computers \& structures 49 (5) (1993) 885-896.

[58] G. I. Rozvany, A critical review of established methods of structural topology optimization, Structural and multidisciplinary optimization 37 (3) (2009) 217237.

[59] N. P. van Dijk, K. Maute, M. Langelaar, F. Van Keulen, Level-set methods for structural topology optimization: a review, Structural and Multidisciplinary Optimization 48 (3) (2013) 437-472.

[60] J.-H. Zhu, W.-H. Zhang, L. Xia, Topology optimization in aircraft and aerospace structures design, Archives of Computational Methods in Engineering 23 (4) (2016) 595-622.

[61] J. D. Deaton, R. V. Grandhi, A survey of structural and multidisciplinary continuum topology optimization: post 2000, Structural and Multidisciplinary Optimization 49 (1) (2014) 1-38.

[62] J. Ganghoffer, I. Goda, A. Novotny, R. Rahouadj, J. Sokolowski, Homogenized couple stress model of optimal auxetic microstructures computed by topology optimization, ZAMM-Journal of Applied Mathematics and Mechanics/Zeitschrift für Angewandte Mathematik und Mechanik 98 (5) (2018) 696-717.

[63] J. Yvonnet, X. Chen, P. Sharma, Apparent flexoelectricity due to heterogeneous piezoelectricity, Journal of Applied Mechanics 87 (11) (2020) 111003.

[64] M. Gologanu, J.-B. Leblond, G. Perrin, J. Devaux, Recent Extensions of Gurson's Model for Porous Ductile Metals, Springer Vienna, Vienna, 1997, pp. 61-130. doi:10.1007/978-3-7091-2662-2_2. 
[65] S. Forest, F. Pradel, K. Sab, Asymptotic analysis of heterogeneous cosserat media, International Journal of Solids and Structures 38 (26) (2001) 4585 - 4608. doi: $10.1016 / \mathrm{S} 0020-7683(00) 00295-\mathrm{X}$

[66] J. Yvonnet, N. Auffray, V. Monchiet, Computational second-order homogenization of materials with effective anisotropic strain-gradient behavior, International Journal of Solids and Structures (2020). doi:10.1016/j . ijsolstr.2020.01. 006.

[67] X. Tian, M. Xu, Q. Deng, J. Sladek, V. Sladek, M. Repka, Q. Li, Sizedependent direct and converse flexoelectricity around a micro-hole, Acta Mechanica 231 (12) (2020) 4851-4865.

[68] Z. Shen, W. Chen, Converse flexoelectric effect in comb electrode piezoelectric microbeam, Physics Letters A 376 (19) (2012) 1661-1663.

[69] N. Mawassy, H. Reda, J.-F. Ganghoffer, V. A. Eremeyev, H. Lakiss, A variational approach of homogenization of piezoelectric composites towards piezoelectric and flexoelectric effective media, International Journal of Engineering Science 158 (2021) 103410.

[70] J. Arrison, Z. Ounaies, Piezoelectric polymers encyclopedia of polymer science and technology (2002).

[71] M. P. Bendsøe, Optimal shape design as a material distribution problem, Structural optimization 1 (4) (1989) 193-202.

[72] G. I. Rozvany, M. Zhou, T. Birker, Generalized shape optimization without homogenization, Structural optimization 4 (3-4) (1992) 250-252.

[73] M. P. Bendsøe, O. Sigmund, Material interpolation schemes in topology optimization, Archive of applied mechanics 69 (9-10) (1999) 635-654.

[74] J. E. Kim, D. S. Kim, P. S. Ma, Y. Y. Kim, Multi-physics interpolation for the topology optimization of piezoelectric systems, Computer Methods in Applied 
Mechanics and Engineering 199 (49) (2010) 3153 - 3168. doi:10.1016/j. cma.2010.06.021

[75] K. Svanberg, A class of globally convergent optimization methods based on conservative convex separable approximations, SIAM Journal on Optimization 12 (2) (2002) 555-573. doi:10.1137/S1052623499362822.

[76] V. Komkov, K. Choi, E. Haug, Design Sensitivity Analysis of Structural Systems, Mathematics in science and engineering, Elsevier Science, 1986.

[77] M. P. Bendsøe, O. Sigmund, Topology Optimization Theory, Methods, and Applications-second edition, 2013.

[78] R. Brenner, Numerical computation of the response of piezoelectric composites using fourier transform, Physical Review B 79 (18) (2009) 184106.

[79] K. S. Ramadan, D. Sameoto, S. Evoy, A review of piezoelectric polymers as functional materials for electromechanical transducers, Smart Materials and Structures 23 (3) (2014) 033001.

[80] W. Ma, L. Cross, Flexoelectricity of barium titanate, Applied Physics Letters $88(23)(2006) 232902$.

[81] S. Forest, D. Trinh, Generalized continua and non-homogeneous boundary conditions in homogenisation methods, ZAMM-Journal of Applied Mathematics and Mechanics/Zeitschrift für Angewandte Mathematik und Mechanik 91 (2) (2011) 90-109.

[82] J. Li, X.-B. Zhang, A numerical approach for the establishment of strain gradient constitutive relations in periodic heterogeneous materials, European Journal of Mechanics-A/Solids 41 (2013) 70-85.

[83] V. Monchiet, N. Auffray, J. Yvonnet, Strain-gradient homogenization: a bridge between asymptotic expansion and quadratic boundary condition methods, Submitted. 
[84] J. Yvonnet, N. Auffray, V. Monchiet, Computational second-order homogenization of materials with effective anisotropic strain gradient behavior, International Journal of Solids and Structures 191-192 (2020) 434-448. 